Geometry 85 Topology

Volume 7 (2003) 255-286

Published: 31 March 2003

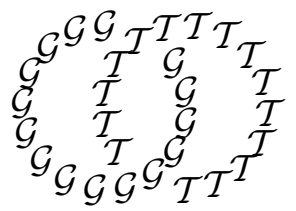

\title{
Manifolds with non-stable fundamental groups at infinity, II
}

\author{
C R Guilbault \\ F C Tinsley \\ Department of Mathematical Sciences, University of Wisconsin-Milwaukee \\ Milwaukee, Wisconsin 53201, USA \\ and \\ Department of Mathematics, The Colorado College \\ Colorado Springs, Colorado 80903, USA \\ Email: craigg@uwm.edu, ftinsley@cc.colorado.edu
}

\begin{abstract}
In this paper we continue an earlier study of ends non-compact manifolds. The over-arching goal is to investigate and obtain generalizations of Siebenmann's famous collaring theorem that may be applied to manifolds having non-stable fundamental group systems at infinity. In this paper we show that, for manifolds with compact boundary, the condition of inward tameness has substatial implications for the algebraic topology at infinity. In particular, every inward tame manifold with compact boundary has stable homology (in all dimensions) and semistable fundamental group at each of its ends. In contrast, we also construct examples of this sort which fail to have perfectly semistable fundamental group at infinity. In doing so, we exhibit the first known examples of open manifolds that are inward tame and have vanishing Wall finiteness obstruction at infinity, but are not pseudo-collarable.
\end{abstract}

\section{AMS Classification numbers Primary: 57N15, 57Q12}

Secondary: 57R65, 57Q10

Keywords: End, tame, inward tame, open collar, pseudo-collar, semistable, Mittag-Leffler, perfect group, perfectly semistable, Z-compactification

Proposed: Steve Ferry

Seconded: Benson Farb, Robion Kirby
Received: 6 September 2002

Accepted: 12 March 2003 


\section{Introduction}

In [7] we presented a program for generalizing Siebenmann's famous collaring theorem (see [15]) to include open manifolds with non-stable fundamental group systems at infinity. To do this, it was first necessary to generalize the notion of an open collar. Define a manifold $N^{n}$ with compact boundary to be a homotopy collar provided $\partial N^{n} \hookrightarrow N^{n}$ is a homotopy equivalence. Then define a pseudo-collar to be a homotopy collar which contains arbitrarily small homotopy collar neighborhoods of infinity. An open manifold is pseudo-collarable if it contains a pseudo-collar neighborhood of infinity. The main results of our initial investigation may be summarized as follows:

Theorem 1.1 (see [7]) Let $M^{n}$ be a one ended $n$-manifold with compact (possibly empty) boundary. If $M^{n}$ is pseudo-collarable, then

(1) $M^{n}$ is inward tame at infinity,

(2) $\pi_{1}\left(\varepsilon\left(M^{n}\right)\right)$ is perfectly semistable, and

(3) $\sigma_{\infty}\left(M^{n}\right)=0 \in \widetilde{K}_{0}\left(\pi_{1}\left(\varepsilon\left(M^{n}\right)\right)\right)$.

Conversely, for $n \geq 7$, if $M^{n}$ satisfies conditions ((1)-(3) and $\pi_{2}\left(\varepsilon\left(M^{n}\right)\right)$ is semistable, then $M^{n}$ is pseudo-collarable.

Remark 1 While it its convenient (and traditional) to focus on one ended manifolds, this theorem actually applies to all manifolds with compact boundary -in particular, to all open manifolds. The key here is that an inward tame manifold with compact boundary has only finitely many ends - we provide a proof of this fact in Section 3. Hence, Theorem 1.1 may be applied to each end individually. For manifolds with non-compact boundaries, the situation is quite different. A straight forward infinite-ended example of this type is given in Section 3. A more detailed discussion of manifolds with non-compact boundaries will be provided in [9].

The condition of inward tameness means (informally) that each neighborhood of infinity can be pulled into a compact subset of itself. We let $\pi_{1}\left(\varepsilon\left(M^{n}\right)\right)$ denote the inverse system of fundamental groups of neighborhoods of infinity. Such a system is semistable if it is equivalent to a system in which all bonding maps are surjections. If, in addition, it can be arranged that the kernels of these bonding maps are perfect groups, then the system is perfectly semistable. The obstruction $\sigma_{\infty}\left(M^{n}\right) \in \widetilde{K}_{0}\left(\pi_{1}\left(\varepsilon\left(M^{n}\right)\right)\right)$ vanishes precisely when each (clean) neighborhood of infinity has finite homotopy type. More precise formulations of 
these definitions are given in Section 2. For a detailed discussion of the structure of pseudo-collars, along with some useful examples of pseudo-collarable and non-pseudo-collarable manifolds, the reader is referred to Section 4 of [7].

One obvious question suggested by Theorem 1.1 is whether the $\pi_{2}$-semistability condition can be omitted from the converse, ie, whether conditions (1)-(3) are sufficient to guarantee pseudo-collarability. We are not yet able to resolve that issue. In this paper, we focus on other questions raised in [7]. The first asks whether inward tameness implies $\pi_{1}$-semistability; and the second asks whether inward tameness (possibly combined with condition 3)) guarantees perfect semistability of $\pi_{1}$. Thus, one arrives at the question: Are conditions (1) and (3) sufficient to ensure pseudo-collarability? Some motivation for this last question is provided by [3] where it is shown that these conditions do indeed characterize pseudo-collarability in Hilbert cube manifolds.

Our first main result provides a positive answer to the $\pi_{1}$-semistability question, and more. It shows that - for manifolds with compact boundary - the inward tameness hypothesis, by itself, has significant implications for the algebraic topology of that manifold at infinity.

Theorem 1.2 If an $n$-manifold with compact (possibly empty) boundary is inward tame at infinity, then it has finitely many ends, each of which has semistable fundamental group and stable homology in all dimensions.

Our second main result provides a negative answer to the pseudo-collarability question discussed above.

Theorem 1.3 For $n \geq 6$, there exists a one ended open $n$-manifold $M_{*}^{n}$ in which all clean neighborhoods of infinity have finite homotopy types (hence, $M_{*}^{n}$ satisfies conditions (1) and (3) from above), but which does not have perfectly semistable fundamental group system at infinity. Thus, $M_{*}^{n}$ is not pseudocollarable.

Theorems 1.2 and 1.3 and their proofs are independent. The first is a very general result that is valid in all dimensions. Its proof is contained in Section 3 . The second involves the construction of rather specific high-dimensional examples, with a blueprint being provided by a significant dose of combinatorial group theory. Although independent, Theorem 1.2 offers crucial guidance on how delicate such a construction must be. The necessary group theory and the construction of the examples may be found in Section 4. Section 2 contains 
the background and definitions needed to read each of the above. In the final section of this paper we discuss a related open question.

The authors wish to acknowledge Tom Thickstun for some very helpful discussions.

The first author wishes to acknowledge support from NSF Grant DMS-0072786.

\section{Definitions and Background}

This section contains most of the terminology and notation needed in the remainder of the paper. It is divided into two subsections - the first devoted to inverse sequences of groups, and the second to the topology of ends of manifolds.

\subsection{Algebra of inverse sequences}

Throughout this section all arrows denote homomorphisms, while arrows of the type $\rightarrow$ or $\nleftarrow$ denote surjections. The symbol $\cong$ denotes isomorphisms.

Let

$$
G_{0} \stackrel{\lambda_{1}}{\longleftarrow} G_{1} \stackrel{\lambda_{2}}{\longleftarrow} G_{2} \stackrel{\lambda_{3}}{\longleftarrow} \ldots
$$

be an inverse sequence of groups and homomorphisms. A subsequence of $\left\{G_{i}, \lambda_{i}\right\}$ is an inverse sequence of the form:

$$
G_{i_{0}} \stackrel{\lambda_{i_{0}+1^{\circ \cdots} \cdots \lambda_{i_{1}}}^{\longleftarrow}}{\longleftarrow} G_{i_{1}} \stackrel{\lambda_{i_{1}+1} \circ \cdots \circ \lambda_{i_{2}}}{\longleftarrow} G_{i_{2}} \stackrel{\lambda_{i_{2}+1^{\circ} \cdots \circ} \lambda_{i_{3}}}{\longleftarrow} \ldots
$$

In the future we will denote a composition $\lambda_{i} \circ \cdots \circ \lambda_{j}(i \leq j)$ by $\lambda_{i, j}$.

We say that sequences $\left\{G_{i}, \lambda_{i}\right\}$ and $\left\{H_{i}, \mu_{i}\right\}$ are pro-equivalent if, after passing to subsequences, there exists a commuting diagram:

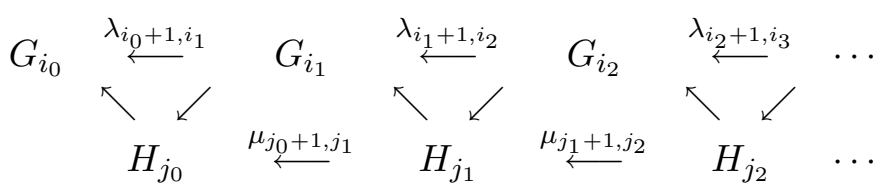

Clearly an inverse sequence is pro-equivalent to any of its subsequences. To avoid tedious notation, we often do not distinguish $\left\{G_{i}, \lambda_{i}\right\}$ from its subsequences. Instead we simply assume that $\left\{G_{i}, \lambda_{i}\right\}$ has the desired properties of a preferred subsequence - often prefaced by the words "after passing to a subsequence and relabelling". 
The inverse limit of a sequence $\left\{G_{i}, \lambda_{i}\right\}$ is a subgroup of $\prod G_{i}$ defined by

$$
\lim _{\longleftarrow}\left\{G_{i}, \lambda_{i}\right\}=\left\{\left(g_{0}, g_{1}, g_{2}, \cdots\right) \in \prod_{i=0}^{\infty} G_{i} \mid \lambda_{i}\left(g_{i}\right)=g_{i-1}\right\} .
$$

Notice that for each $i$, there is a projection homomorphism $p_{i}: \lim _{\longleftarrow}\left\{G_{i}, \lambda_{i}\right\} \rightarrow$ $G_{i}$. It is a standard fact that pro-equivalent inverse sequences have isomorphic inverse limits.

An inverse sequence $\left\{G_{i}, \lambda_{i}\right\}$ is stable if it is pro-equivalent to an inverse sequence $\left\{H_{i}, \mu_{i}\right\}$ for which each $\mu_{i}$ is an isomorphism. Equivalently, $\left\{G_{i}, \lambda_{i}\right\}$ is stable if, after passing to a subsequence and relabelling, there is a commutative diagram of the form

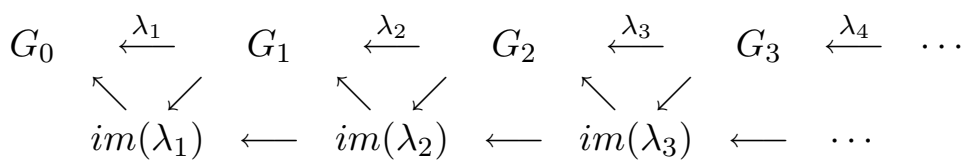

where each bonding map in the bottom row (obtained by restricting the corresponding $\left.\lambda_{i}\right)$ is an isomorphism. If $\left\{H_{i}, \mu_{i}\right\}$ can be chosen so that each $\mu_{i}$ is an epimorphism, we say that our inverse sequence is semistable (or Mittag-Leffler, or pro-epimorphic). In this case, it can be arranged that the restriction maps in the bottom row of $(*)$ are epimorphisms. Similarly, if $\left\{H_{i}, \mu_{i}\right\}$ can be chosen so that each $\mu_{i}$ is a monomorphism, we say that our inverse sequence is promonomorphic; it can then be arranged that the restriction maps in the bottom row of $(*)$ are monomorphisms. It is easy to see that an inverse sequence that is semistable and pro-monomorphic is stable.

Recall that a commutator element of a group $H$ is an element of the form $x^{-1} y^{-1} x y$ where $x, y \in H$; and the commutator subgroup of $H$, denoted $[H, H]$, is the subgroup generated by all of its commutators. The group $H$ is perfect if $[H, H]=H$. An inverse sequence of groups is perfectly semistable if it is pro-equivalent to an inverse sequence

$$
G_{0} \stackrel{\lambda_{1}}{\longleftarrow} G_{1} \stackrel{\lambda_{2}}{\longleftarrow} G_{2} \stackrel{\lambda_{3}}{\longleftarrow} \cdots
$$

of finitely presentable groups and surjections where each ker $\left(\lambda_{i}\right)$ perfect. The following shows that inverse sequences of this type behave well under passage to subsequences.

Lemma 2.1 A composition of surjective group homomorphisms, each having perfect kernels, has perfect kernel. Thus, if an inverse sequence of surjective group homomorphisms has the property that the kernel of each bonding map is perfect, then each of its subsequences also has this property. 
Proof See Lemma 1 of [7].

For later use, we record an easy but crucial property of perfect groups.

Lemma 2.2 If $f: G \rightarrow H$ is a surjective group homomorphism and $G$ is perfect, then $H$ is perfect.

Proof The image of each commutator from $G$ is a commutator in $H$.

We conclude this section with a technical result that will be needed later. Compare to the well-known Five Lemma from homological algebra.

Lemma 2.3 Assume the following commutative diagram of five inverse sequences:

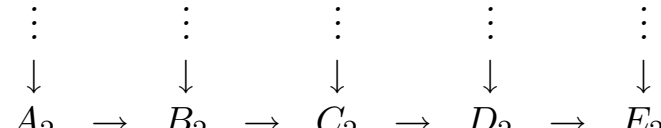

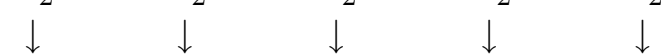

$$
\begin{aligned}
& A_{1} \rightarrow B_{1} \rightarrow C_{1} \rightarrow D_{1} \rightarrow E_{1} \\
& \downarrow \quad \downarrow \quad \downarrow \quad \downarrow \quad \downarrow \quad \downarrow \quad \downarrow \\
& A_{0} \rightarrow B_{0} \rightarrow C_{0} \rightarrow D_{0} \rightarrow E_{0}
\end{aligned}
$$

If each row is exact and the inverse sequences $\left\{A_{i}\right\},\left\{B_{i}\right\},\left\{D_{i}\right\}$, and $\left\{E_{i}\right\}$ are stable, then so is $\left\{C_{i}\right\}$.

Proof The proof is by an elementary but intricate diagram chase. See Lemmas 2.1 and 2.2 of $[6]$.

\subsection{Topology of ends of manifolds}

In this paper, the term manifold means manifold with (possibly empty) boundary. A manifold is open if it is non-compact and has no boundary. For convenience, all manifolds are assumed to be PL. Analogous results may be obtained for smooth or topological manifolds in the usual ways.

Let $M^{n}$ be a manifold with compact (possibly empty) boundary. A set $N \subset M^{n}$ is a neighborhood of infinity if $\overline{M^{n}-N}$ is compact. A neighborhood of infinity $N$ is clean if

- $\quad N$ is a closed subset of $M^{n}$, 
- $\quad N \cap \partial M^{n}=\emptyset$, and

- $N$ is a codimension 0 submanifold of $M^{n}$ with bicollared boundary.

It is easy to see that each neighborhood of infinity contains a clean neighborhood of infinity.

Remark 2 We have taken advantage of the compact boundary by requiring that clean neighborhoods of infinity be disjoint from $\partial M^{n}$. In the case of non-compact boundary, a slightly more delicate definition is required.

We say that $M^{n}$ has $k$ ends if it contains a compactum $C$ such that, for every compactum $D$ with $C \subset D, M^{n}-D$ has exactly $k$ unbounded components, ie, $k$ components with noncompact closures. When $k$ exists, it is uniquely determined; if $k$ does not exist, we say $M^{n}$ has infinitely many ends.

If $M^{n}$ has compact boundary and is $k$-ended, then $M^{n}$ contains a clean neighborhood of infinity $N$ that consists of $k$ connected components, each of which is a one ended manifold with compact boundary. Therefore, when studying manifolds (or other spaces) having finitely many ends, it suffices to understand the one ended situation. In this paper, we are primarily concerned with manifolds possessing finitely many ends (See Theorem 1.2 or Prop. 3.1), and thus, we frequently restrict our attention to the one ended case.

A connected clean neighborhood of infinity with connected boundary is called a 0-neighborhood of infinity. If $N$ is clean and connected but has more than one boundary component, we may choose a finite collection of disjoint properly embedded arcs in $N$ that connect these components. Deleting from $N$ the interiors of regular neighborhoods of these arcs produces a 0 -neighborhood of infinity $N_{0} \subset N$.

A nested sequence $N_{0} \supset N_{1} \supset N_{2} \supset \cdots$ of neighborhoods of infinity is cofinal if $\bigcap_{i=0}^{\infty} N_{i}=\emptyset$. For any one ended manifold $M^{n}$ with compact boundary, one may easily obtain a cofinal sequence of 0 -neighborhoods of infinity.

We say that $M^{n}$ is inward tame at infinity if, for arbitrarily small neighborhoods of infinity $N$, there exist homotopies $H: N \times[0,1] \rightarrow N$ such that $H_{0}=i d_{N}$ and $\overline{H_{1}(N)}$ is compact. Thus inward tameness means each neighborhood of infinity can be pulled into a compact subset of itself. In this situation, the $H$ 's will be referred to as taming homotopies.

Recall that a complex $X$ is finitely dominated if there exists a finite complex $K$ and maps $u: X \rightarrow K$ and $d: K \rightarrow X$ such that $d \circ u \simeq i d_{X}$. The following lemma uses this notion to offer equivalent formulations of "inward tameness". 
Lemma 2.4 For a manifold $M^{n}$, the following are equivalent.

(1) $M^{n}$ is inward tame at infinity.

(2) Each clean neighborhood of infinity in $M^{n}$ is finitely dominated.

(3) For each cofinal sequence $\left\{N_{i}\right\}$ of clean neighborhoods of infinity, the inverse sequence

$$
N_{0} \stackrel{j_{1}}{\hookleftarrow} N_{1} \stackrel{j_{2}}{\hookleftarrow} N_{2} \stackrel{j_{3}}{\hookleftarrow} \ldots
$$

is pro-homotopy equivalent to an inverse sequence of finite polyhedra.

Proof To see that (1) implies (2), let $N$ be a clean neighborhood of infinity and $H: N \times[0,1] \rightarrow N$ a taming homotopy. Let $K$ be a polyhedral subset of $N$ that contains $\overline{H_{1}(N)}$. If $u: N \rightarrow K$ is obtained by restricting the range of $H_{1}$ and $d: K \hookrightarrow N$, then $d \circ u=H_{1} \simeq i d_{N}$, so $N$ is finitely dominated.

To see that 2) implies 3), choose for each $N_{i}$ a finite polyhedron $K_{i}$ and maps $u_{i}: N_{i} \rightarrow K_{i}$ and $d_{i}: K_{i} \rightarrow N_{i}$ such that $d_{i} \circ u_{i} \simeq i d_{N_{i}}$. For each $i \geq 1$, let $f_{i}=u_{i-1} \circ j_{i}$ and $g_{i}=f_{i} \circ d_{i}$. Since $d_{i-1} \circ f_{i}=d_{i-1} \circ u_{i-1} \circ j_{i} \simeq i d_{N_{i-1}} \circ j_{i}=j_{i}$, the diagram

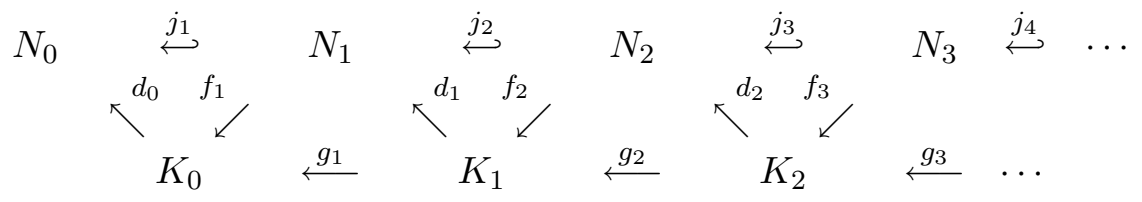

commutes up to homotopy, so (by definition) the two inverse sequences are pro-homotopy equivalent.

Lastly, we assume the existence of a homotopy commutative diagram as pictured above for some cofinal sequence of clean neighborhoods of infinity and some inverse sequence of finite polyhedra. We show that for each $i \geq 1$, there is a taming homotopy for $N_{i}$. By hypothesis, $d_{i} \circ f_{i+1} \simeq j_{i+1}$. Extend $j_{i+1}$ to $i d_{N_{i}}$, then apply the homotopy extension property (see [10, pp.14-15]) for the pair $\left(N_{i}, N_{i+1}\right)$ to obtain $H: N_{i} \times[0,1] \rightarrow N_{i}$ with $H_{0}=i d_{N_{i}}$ and $\left.H_{1}\right|_{N_{i+1}}=$ $d_{i} \circ f_{i+1}$. Now,

$$
H_{1}\left(N_{i}\right)=H_{1}\left(N_{i}-N_{i+1}\right) \cup H_{1}\left(N_{i+1}\right) \subset H_{1}\left(\overline{N_{i}-N_{i+1}}\right) \cup d_{i}\left(K_{i}\right),
$$

so $\overline{H_{1}\left(N_{i}\right)}$ is compact, and $H$ is the desired taming homotopy.

Given a nested cofinal sequence $\left\{N_{i}\right\}_{i=0}^{\infty}$ of connected neighborhoods of infinity, base points $p_{i} \in N_{i}$, and paths $\alpha_{i} \subset N_{i}$ connecting $p_{i}$ to $p_{i+1}$, we obtain an inverse sequence:

$$
\pi_{1}\left(N_{0}, p_{0}\right) \stackrel{\lambda_{1}}{\longleftarrow} \pi_{1}\left(N_{1}, p_{1}\right) \stackrel{\lambda_{2}}{\longleftarrow} \pi_{1}\left(N_{2}, p_{2}\right) \stackrel{\lambda_{3}}{\longleftarrow} \cdots
$$


Here, each $\lambda_{i+1}: \pi_{1}\left(N_{i+1}, p_{i+1}\right) \rightarrow \pi_{1}\left(N_{i}, p_{i}\right)$ is the homomorphism induced by inclusion followed by the change of base point isomorphism determined by $\alpha_{i}$. The obvious singular ray obtained by piecing together the $\alpha_{i}$ 's is often referred to as the base ray for the inverse sequence. Provided the sequence is semistable, one can show that its pro-equivalence class does not depend on any of the choices made above. We refer to the pro-equivalence class of this sequence as the fundamental group system at infinity for $M^{n}$ and denote it by $\pi_{1}\left(\varepsilon\left(M^{n}\right)\right)$. (In the absence of semistability, the pro-equivalence class of the inverse sequence depends on the choice of base ray, and hence, this choice becomes part of the data.) It is easy to see how the same procedure may also be used to define $\pi_{k}\left(\varepsilon\left(M^{n}\right)\right)$ for $k>1$.

For any coefficient ring $R$ and any integer $j \geq 0$, a similar procedure yields an inverse sequence

$$
H_{j}\left(N_{0} ; R\right) \stackrel{\lambda_{1}}{\longleftarrow} H_{j}\left(N_{1} ; R\right) \stackrel{\lambda_{2}}{\longleftarrow} H_{j}\left(N_{2} ; R\right) \stackrel{\lambda_{3}}{\longleftarrow} \cdots
$$

where each $\lambda_{i}$ is induced by inclusion - here, no base points or rays are needed. We refer to the pro-equivalence class of this sequence as the $j^{\text {th }}$ homology at infinity for $M^{n}$ with $R$-coefficients and denote it by $H_{j}\left(\varepsilon\left(M^{n}\right) ; R\right)$.

In [17], Wall shows that each finitely dominated connected space $X$ determines a well-defined element $\sigma(X)$ lying in $\widetilde{K}_{0}\left(\mathbb{Z}\left[\pi_{1} X\right]\right.$ ) (the group of stable equivalence classes of finitely generated projective $\mathbb{Z}\left[\pi_{1} X\right]$-modules under the operation induced by direct sum) that vanishes if and only if $X$ has the homotopy type of a finite complex. Given a nested cofinal sequence $\left\{N_{i}\right\}_{i=0}^{\infty}$ of connected clean neighborhoods of infinity in an inward tame manifold $M^{n}$, we have a Wall obstruction $\sigma\left(N_{i}\right)$ for each $i$. These may be combined into a single obstruction

$$
\begin{aligned}
\sigma_{\infty}\left(M^{n}\right) & =(-1)^{n}\left(\sigma\left(N_{0}\right), \sigma\left(N_{1}\right), \sigma\left(N_{2}\right), \cdots\right) \\
& \in \widetilde{K}_{0}\left(\pi_{1}\left(\varepsilon\left(M^{n}\right)\right)\right) \equiv \varliminf_{\varlimsup} \widetilde{K}_{0}\left(\mathbb{Z}\left[\pi_{1} N_{i}\right]\right)
\end{aligned}
$$

that is well-defined and which vanishes if and only if each clean neighborhood of infinity in $M^{n}$ has finite homotopy type. See [3] for details.

We close this section with a known result from the topology of manifolds. Its proof is short and its importance is easily seen when one considers the "onesided $h$-cobordism" ( $\left.W, \partial N, \partial N^{\prime}\right)$ that occurs naturally when $N^{\prime}$ is a homotopy collar contained in the interior of another homotopy collar $N$ and $W=\overline{N-N^{\prime}}$. In particular, this result explains why pseudo-collarable manifolds must have perfectly semistable fundamental groups at their ends. Additional details may be found in Section 4 of [7]. 
Theorem 2.5 Let $\left(W^{n}, P, Q\right)$ be a compact connected cobordism between closed $(n-1)$-manifolds with the property that $P \hookrightarrow W^{n}$ is a homotopy equivalence. Then the inclusion induced map $i_{\#}: \pi_{1}(Q) \rightarrow \pi_{1}\left(W^{n}\right)$ is surjective and has perfect kernel.

Proof Let $p: \widetilde{W} \rightarrow W^{n}$ be the universal covering projection, $\widetilde{P}=p^{-1}(P)$, and $\widehat{Q}=p^{-1}(Q)$. By Poincaré duality for non-compact manifolds,

$$
H_{k}(\widetilde{W}, \widehat{Q} ; \mathbb{Z}) \cong H_{c}^{n-k}(\widetilde{W}, \widetilde{P} ; \mathbb{Z})
$$

where cohomology is with compact supports. Since $\widetilde{P} \hookrightarrow \widetilde{W}$ is a proper homotopy equivalence, all of these relative cohomology groups vanish. It follows that $H_{1}(\widetilde{W}, \widehat{Q} ; \mathbb{Z})=0$, so by the long exact sequence for $(\widetilde{W}, \widehat{Q}), \widetilde{H}_{0}(\widehat{Q} ; \mathbb{Z})=0$; therefore $\widehat{Q}$ is connected. By covering space theory, the components of $\widehat{Q}$ are in one-to-one correspondence with the cosets of $i_{\#}\left(\pi_{1}(Q)\right)$ in $\pi_{1}\left(W^{n}\right)$, so $i_{\#}$ is surjective. Similarly, $H_{2}(\widetilde{W}, \widehat{Q} ; \mathbb{Z})=0$, and since $\widetilde{W}$ is simply connected, the long exact sequence for $(\widetilde{W}, \widehat{Q})$ shows that $H_{1}(\widehat{Q} ; \mathbb{Z})=0$. This implies that $\pi_{1}(\widehat{Q})$ is a perfect group, and covering space theory tell us that $\pi_{1}(\widehat{Q}) \cong \operatorname{ker}\left(i_{\#}\right)$.

\section{Inward tameness, $\pi_{1}$-semistability, and $H_{*}$-stability}

The theme of this section is that - for manifolds with compact (possibly empty) boundary-inward tameness, by itself, has some significant consequences. In particular, an inward tame manifold of this type has:

- finitely many ends,

- semistable fundamental group at each of these ends, and

- stable (finitely generated) homology at infinity in all dimensions.

The first of these properties is known; for completeness, we will provide a proof. The second property answers a question posed in [7]. A stronger conclusion of $\pi_{1}$-stability is not possible, as can be seen in the exotic universal covering spaces constructed in [5]. (See Example 3 of [7] for a discussion.) Somewhat surprisingly, inward tameness does imply stability at infinity for homology in the situation at hand. 
It is worth noting that, under slightly weaker hypotheses, none of these properties holds. We provide some simple examples of locally finite complexes, and polyhedral manifolds (with non-compact boundaries) that violate each of the above.

Example 1 Let $E$ denote a wedge of two circles. Then the universal cover $\widetilde{E}$ of $E$ is an inward tame 1-complex with infinitely many ends.

Example 2 Let $f:\left(S^{1}, *\right) \rightarrow\left(S^{1}, *\right)$ be degree 2 map, and let $X$ be the "inverse mapping telescope" of the system:

$$
S^{1} \stackrel{f}{\longleftarrow} S^{1} \stackrel{f}{\longleftarrow} S^{1} \stackrel{f}{\longleftarrow} \cdots
$$

Assemble a base ray from the mapping cylinder arcs corresponding to the base point $*$. It is easy to see that $X$ is inward tame and that $\pi_{1}(\varepsilon(X))$ is represented by the system

$$
\mathbb{Z} \stackrel{\times 2}{\longleftarrow} \mathbb{Z} \stackrel{\times 2}{\longleftarrow} \mathbb{Z} \stackrel{\times 2}{\longleftarrow} \cdots
$$

which is not semistable. Hence, $\pi_{1}$-semistability does not follow from inward tameness for one ended complexes. This example also shows that inward tame complexes needn't have stable $H_{1}(\varepsilon(X) ; \mathbb{Z})$.

Example 3 More generally, if

$$
K_{0} \stackrel{f_{1}}{\longleftarrow} K_{1} \stackrel{f_{2}}{\longleftarrow} K_{3} \stackrel{f_{3}}{\longleftarrow} \cdots
$$

is an inverse sequence of finite polyhedra, then the inverse mapping telescope $Y$ of this sequence is inward tame. By choosing the polyhedra and the bonding maps appropriately, we can obtain virtually any desired behavior in $\pi_{1}(\varepsilon(Y))$ and $H_{k}(\varepsilon(Y) ; \mathbb{Z})$.

Example 4 By properly embedding the above complexes in $\mathbb{R}^{n}$ and letting $M^{n}$ be a regular neighborhood, we may obtain inward tame manifold examples with similar bad behavior at infinity. Of course, $M^{n}$ will have noncompact boundary.

We are now ready to prove Theorem 1.2. This will be done with a sequence of three propositions - one for each of the bulleted items listed above. The first is the simplest and may be deduced from Theorem 1.10 of [15]. It could also be obtained later, as a corollary of Proposition 3.3. However, Proposition 3.3 and its proof become cleaner if we obtain this result first. The proof is short and rather intuitive. 
Proposition 3.1 Let $M^{n}$ be an $n$-manifold with compact boundary that is inward tame at infinity. Then $M^{n}$ has finitely many ends. More specifically, the number of ends is less than or equal to $\operatorname{rank}\left(H_{n-1}\left(M^{n} ; \mathbb{Z}_{2}\right)\right)+1$. (See the remark below.)

Proof Inward tameness implies that each clean neighborhood of infinity (including $M^{n}$ itself) is finitely dominated and hence, has finitely generated homology in all dimensions. We'll show that $M^{n}$ has at most $k_{0}+1$ ends, where $k_{0}=\operatorname{rank}\left(H_{n-1}\left(M^{n} ; \mathbb{Z}_{2}\right)\right)$.

Let $N$ be an clean neighborhood of infinity, each of whose components is noncompact. Since $H_{0}\left(N ; \mathbb{Z}_{2}\right)$ has finite rank, there are finitely many of these components $\left\{N_{i}\right\}_{i=1}^{p}$. Our theorem follows if we can show that $p$ is bounded by $k_{0}+1$.

Using techniques described in Section 2.2, we may assume that $\partial N_{i}$ is nonempty and connected for all $i$. Then, from the long exact sequence for the pair $\left(N_{i}, \partial N_{i}\right)$, we may deduce that for each $i \operatorname{rank}\left(H_{n-1}\left(N_{i} ; \mathbb{Z}_{2}\right)\right) \geq 1$. Hence, $\operatorname{rank}\left(H_{n-1}\left(N ; \mathbb{Z}_{2}\right)\right) \geq p$

Let $C=\overline{M^{n}-N}$. Then $C$ is a compact codimension 0 submanifold of $M^{n}$, and its boundary consists of the disjoint union of $\partial M^{n}$ with $\partial N$. Thus, $\operatorname{rank}\left(H_{n-1}\left(\partial C ; \mathbb{Z}_{2}\right)\right)=p+q$, where $q$ is the number of components in $\partial M^{n}$. From the long exact sequence for the pair $(C, \partial C)$ we may conclude that $\operatorname{rank}\left(H_{n-1}\left(C ; \mathbb{Z}_{2}\right)\right) \geq p+q-1$.

Now consider the following Mayer-Vietoris sequence:

$$
\begin{aligned}
& \rightarrow H_{n-1}\left(\partial N ; \mathbb{Z}_{2}\right) \rightarrow H_{n-1}\left(C ; \mathbb{Z}_{2}\right) \oplus H_{n-1}\left(N ; \mathbb{Z}_{2}\right) \quad \rightarrow \quad H_{n-1}\left(M^{n} ; \mathbb{Z}_{2}\right) \rightarrow \\
& \bigoplus_{i=1}^{p} \mathbb{Z}_{2} \\
& \bigoplus_{i=1}^{k_{0}} \mathbb{Z}_{2}
\end{aligned}
$$

Since $\mathbb{Z}_{2}$ is a field, exactness implies that the rank of the middle term is no greater than the sum of the ranks of the first and third terms. The first summand of the middle term has rank $\geq p+q-1$ and the second summand has rank $\geq p$. Hence $2 p+q-1 \leq p+k_{0}$. It follows that $p \leq k_{0}+1$.

Remark 3 The number of ends of $M^{n}$ may be less than $\operatorname{rank}\left(H_{n-1}\left(M^{n} ; \mathbb{Z}_{2}\right)\right)$ +1 . Indeed, by "connect summing" copies of $S^{n-1} \times S^{1}$ to $\mathbb{R}^{n}$, one can make the difference between these numbers arbitrarily large. The issue is that some generators of $H_{n-1}\left(M^{n} ; \mathbb{Z}_{2}\right)$ do not "split off an end". To obtain strict equality one should add 1 to the rank of the kernel of

$$
\lambda: H_{n-1}\left(M^{n} ; \mathbb{Z}_{2}\right) \rightarrow H_{n-1}^{l f}\left(M^{n} ; \mathbb{Z}_{2}\right)
$$

where $H^{l f}$ denotes homology based on locally finite chains. 
Before proving the remaining two propositions, we fix some notation and describe a "homotopy refinement procedure" that will be applied in each of the proofs. As noted earlier, (by applying Proposition 3.1) it suffices to consider the one ended case, so for the remainder of this section, $M^{n}$ is a one ended inward tame manifold with compact boundary.

Let $\left\{N_{i}\right\}_{i=0}^{\infty}$ be a nested cofinal sequence of 0-neighborhoods of infinity and, for each $i \geq 0$, let $A_{i}=N_{i}-i n t\left(N_{i+1}\right)$. By inward tameness, we may (after passing to a subsequence and relabelling) assume that (for each $i \geq 0$ ) there exists a taming homotopy $H^{i}: N_{i} \times[0,1] \rightarrow N_{i}$ satisfying:

i) $H_{0}^{i}=i d_{N_{i}}$,

ii) $H^{i}$ is fixed on $\partial N_{i}$, and

iii) $H_{1}^{i}\left(N_{i}\right) \subset A_{i}-\partial N_{i+1}$.

Choose a proper embedding $r:[0, \infty) \rightarrow N_{0}$ so that, for each $i, r([i, \infty)) \subset N_{i}$ and so that the image ray $R_{0}$ intersects each $\partial N_{i}$ transversely at the single point $p_{i}=r(i)$. For $i \geq 0$, let $R_{i}=r([i, \infty)) \subset N_{i}$; and let $\alpha_{i}$ denote the arc $r([i, i+1])$ in $A_{i}$ from $p_{i}$ to $p_{i+1}$. In addition, choose an embedding $t: B^{n-1} \times[0, \infty) \rightarrow N_{0}$ whose image $T_{0}$ is a regular neighborhood of $R_{0}$, such that $\left.t\right|_{\{\overline{0}\} \times[0, \infty)}=r$, and so that, for each $i, T_{0}$ intersects $\partial N_{i}$ precisely in the $(n-1)$-disk $D_{i}=t\left(B^{n-1} \times\{i\}\right)$. Let $B^{\prime} \subset \operatorname{int}\left(B^{n-1}\right)$ be an $(n-1)$-ball containing $\overline{0}, T_{0}^{\prime}=t\left(B^{\prime} \times[0, \infty)\right)$ and $D_{i}^{\prime}=t\left(B^{\prime} \times\{i\}\right)$. Then, for each $i \geq 0$,

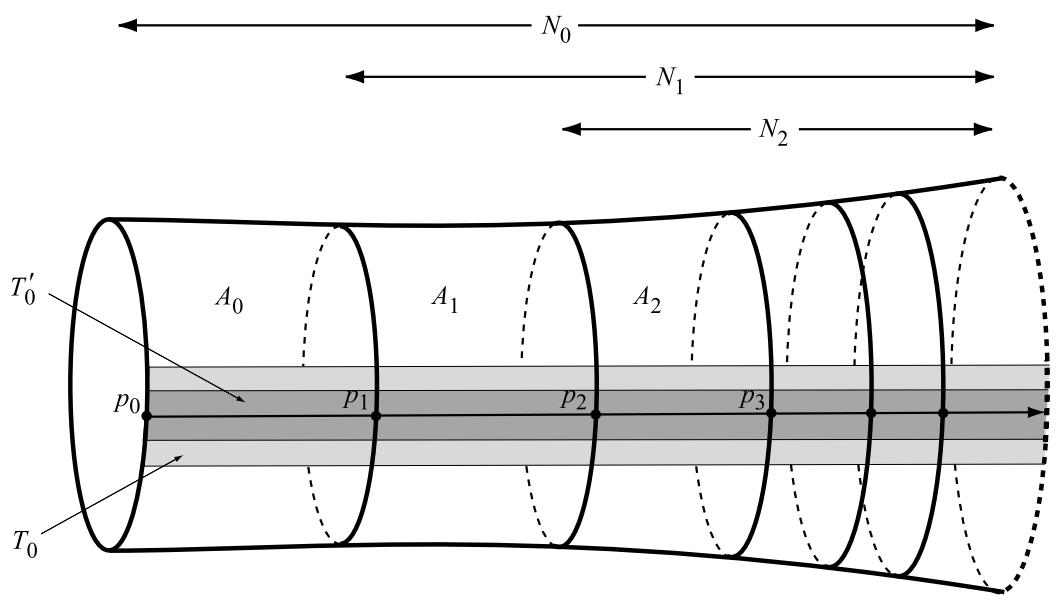

Figure 1

$T_{i}=t\left(B^{n-1} \times[i, \infty)\right)$ and $T_{i}^{\prime}=t\left(B^{\prime} \times[i, \infty)\right)$ are regular neighborhoods of $R_{i}$ in $N_{i}$ intersecting $\partial N_{i}$ in $D_{i}$ and $D_{i}^{\prime}$, respectively. See Figure 1. 
We now show how to refine each $H^{i}$ so that it respects the "base ray" $R_{i}$ and acts in a particularly nice manner on and over $T_{i}^{\prime}$. Let $j^{i}:\left(B^{n-1} \times[i, \infty)\right) \times$ $[0,1] \rightarrow B^{n-1} \times[i, \infty)$ be a strong deformation retraction onto $\partial\left(B^{n-1} \times[i, \infty)\right)$ with the following properties:

a) On $B^{\prime} \times[i, \infty), j^{i}$ is the "radial" deformation retraction onto $B^{\prime} \times\{i\}$ given by $((b, s), u) \mapsto(b, s+u(i-s))$.

b) For $(b, s) \notin B^{\prime} \times[i, \infty)$, the track $j^{i}((b, s) \times[0,1])$ of $(b, s)$ does not intersect $B^{\prime} \times[i, \infty)$.

c) The radial component of each track of $j^{i}$ is non-increasing, ie, if $u_{1} \leq u_{2}$ then $p\left(j^{i}\left(b, s, u_{2}\right)\right) \leq p\left(j^{i}\left(b, s, u_{1}\right)\right)$ where $p$ is projection onto $[i, \infty)$.

Figure 2 represents $j^{i}$, wherein tracks of $j^{i}$ are meant to follow the indicated flow lines.

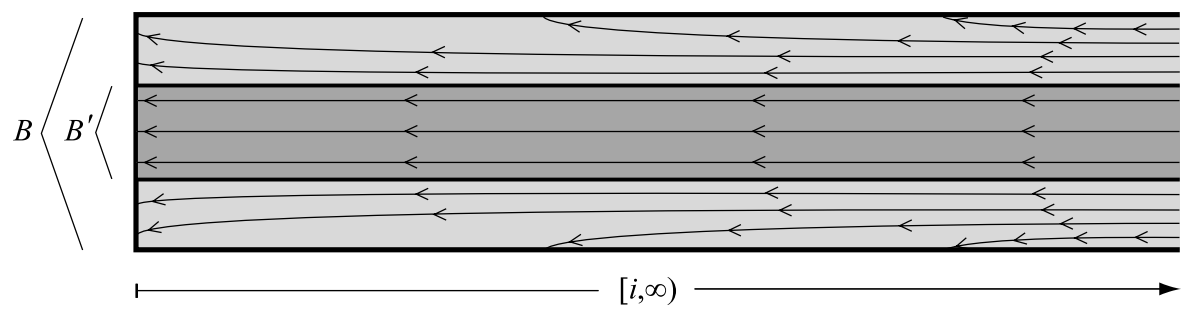

Figure 2

Define $J^{i}: N_{i} \times[0,1] \rightarrow N_{i}$ to be $t \circ j^{i} \circ\left(t^{-1} \times i d\right)$ on $T_{i}$ and the identity outside of $T_{i}$. Then $J^{i}$ is a strong deformation retraction of $N_{i}$ onto $N_{i}-$ $t\left(\stackrel{\circ}{B}^{n-1} \times(i, \infty)\right)$. Define $K^{i}: N_{i} \times[0,1] \rightarrow N_{i}$ as follows:

$$
K^{i}(x, t)=\left\{\begin{array}{cc}
J^{i}(x, 2 t) & 0 \leq t \leq \frac{1}{2} \\
J_{1}^{i}\left(H^{i}\left(J^{i}(x, 1), 2 t-1\right)\right) & \frac{1}{2} \leq t \leq 1
\end{array} .\right.
$$

This homotopy retains the obvious analogs of properties i)-iii). In addition, we have

iv) $K^{i}$ acts in a canonical manner on $T_{i}^{\prime}$, and

v) tracks of points outside of $T_{i}^{\prime}$ do not pass through the interior of $T_{i}^{\prime}$.

Proposition 3.2 Every one ended inward tame $n$-manifold $M^{n}$ with compact boundary has semistable fundamental group at infinity. 
Proof For convenience, assume that $n \geq 3$. For $n=2$ the result may be obtained by applying well-known structure theorems for 2-manifolds, or by modifying our proof slightly.

Let $\left\{N_{i}\right\}_{i=0}^{\infty}$ be a nested cofinal sequence of 0 -neighborhoods of infinity with refined taming homotopies $\left\{K^{i}\right\}_{i=0}^{\infty}$ as constructed above. Other choices and labels are also carried over from above. Note that, for each $i$,

$$
\pi_{1}\left(A_{i}, p_{i}\right) \rightarrow \pi_{1}\left(N_{i}, p_{i}\right) \text { is surjective }
$$

We will show that (for each $i \geq 0$ ) each loop in $N_{i+1}$ based at $p_{i+1}$ can be pushed (rel $\alpha_{i+1}$ ) to a loop in $N_{i+2}$ based at $p_{i+2}$ via a homotopy contained in $N_{i}$. This implies the existence of a diagram of type $(*)$ from section 2 for which the bonding homomorphisms in the bottom row are surjective - and thus, $\pi_{1}$-semistability.

(Note In performing this push, the "rel $\alpha_{i+1}$ " requirement is crucial. The ability to push loops from $N_{i+1}$ into $N_{i+2}$ via a homotopy contained in $N_{i}$ without regards to basepoints - would yield another well-known, but strictly weaker, property called end 1-movability. See [2] for a discussion. Much of the homotopy refinement process described earlier is aimed at obtaining control over the tracks of the base points.)

Let $\tau$ be a chosen loop in $N_{i+1}$ based at $p_{i+1}$. By $(\dagger)$, we may assume that $\tau \subset A_{i+1}-\partial N_{i+2}$. Let $L^{i}: \partial N_{i+2} \times[0,1] \rightarrow N_{i}$ be the restriction of $K^{i}$. Note that $L^{i}\left(\partial N_{i+2} \times\{1\}\right) \subset A_{i}-\partial N_{i+1}$ and that, by condition (iv) above, $L^{i}$ takes $D_{i+2}^{\prime} \times\left[0, \frac{1}{2}\right]$ homeomorphically onto $\overline{T_{i}^{\prime}-T_{i+2}^{\prime}}$ with $D_{i+2}^{\prime} \times\left[\frac{1}{2}, 1\right]$ being flattened onto $D_{i}$. In addition, $L^{i}\left(\left\{p_{i+2}\right\} \times\left[0, \frac{1}{4}\right]\right)=\alpha_{i+1}, L^{i}\left(\left\{p_{i+2}\right\} \times\left[\frac{1}{4}, \frac{1}{2}\right]\right)=$ $\alpha_{i}$, and $L^{i}\left(p_{i+2}, \frac{1}{4}\right)=p_{i+1}$. Without changing its values on $\left(\partial N_{i+2} \times\{0\}\right) \cup$ $\left(D_{i+2}^{\prime} \times\left[0, \frac{1}{2}\right]\right)$, we may adjust $L^{i}$ so that it is a non-degenerate PL mapping. In particular, we may choose triangulations $\Gamma_{1}$ and $\Gamma_{2}$ of the domain and range respectively so that, up to $\varepsilon$-homotopy, $L^{i}$ may be realized as a simplicial map sending each $k$-simplex of $\Gamma_{1}$ onto a $k$-simplex of $\Gamma_{2}$. (See Chapter 5 of [14].) Adjust $\tau$ (rel $\left.p_{i+1}\right)$ so that it is an embedded circle in general position with respect to $\Gamma_{2}$. Then $\left(L^{i}\right)^{-1}(\tau)$ is a closed 1-manifold in $\partial N_{i+2} \times(0,1)$. Let $\sigma$ be the component of $\left(L^{i}\right)^{-1}(\tau)$ containing the point $\left(p_{i+2}, \frac{1}{4}\right)$. Since $L^{i}$ takes a neighborhood of $\left(p_{i+2}, \frac{1}{4}\right)$ homeomorphically onto a neighborhood of $p_{i+1}$, and since no other points of $\sigma$ are taken near $p_{i+1}$ (use condition v) from above), then $L$ restricts to a degree \pm 1 map of $\sigma$ onto $\tau$. Now the natural deformation retraction of $\partial N_{i+2} \times[0,1]$ onto $\partial N_{i+2} \times\{0\}$ pushes $\sigma$ into $\partial N_{i+2} \times\{0\}$ while sliding $\left(p_{i+2}, \frac{1}{4}\right)$ along the arc $\left\{p_{i+2}\right\} \times\left[0, \frac{1}{4}\right]$. Composing this push with $L^{i}$ provides a homotopy of $\tau$ (within $N_{i}$ ) into $\partial N_{i+2}$ whereby $p_{i+1}$ is slid along $\alpha_{i+1}$ to $p_{i+2}$. 
Remark 4 The reader may have noticed that a general principle at work in the proof of Proposition 3.2 is that "degree 1 maps between manifolds induce surjections on fundamental groups". Instead of applying this directly, we used a constructive approach to finding the preimage of a loop. This allowed us to handle orientable and non-orientable cases simultaneously. Proposition 3.3 is based on a similar general principle regarding homology groups and degree 1 maps. However, instead of a unified approach, we first obtain the result for orientable manifolds by applying the general principle directly; then we use the orientable result to extend to the non-orientable case. Those who prefer this approach may use the proof of claim 1 from Proposition 3.3 as an outline to obtain an alternative proof of Proposition 3.2 in the case that $M^{n}$ is orientable.

Proposition 3.3 Let $M^{n}$ be a one ended, inward tame $n$-manifold with compact boundary and let $R$ be a commutative ring with unity. Then $H_{j}\left(\varepsilon\left(M^{n}\right) ; R\right)$ is stable for all $i$.

For the sake of simplicity, we will first prove Proposition 3.3 for $R=\mathbb{Z}$. The more general result will then obtained by an application of the universal coefficient theorem. Alternatively, one could do all of what follows over an arbitrary coefficient ring. Before beginning the proof we review some of the tools needed

Let $W$ be a compact connected orientable $n$-manifold with boundary. Assume that $\partial W=P \cup Q$, where $P$ and $Q$ are disjoint, closed, $(n-1)$-dimensional submanifolds of $\partial W$. We do not require that $P$ or $Q$ be connected or nonempty. Then Poincaré duality tells us that the cap product with an orientation class $[W]$ induces isomorphisms

$$
H^{k}(W, P ; \mathbb{Z}) \stackrel{\cap[W]}{\longrightarrow} H_{n-k}(W, Q ; \mathbb{Z}) .
$$

If $W^{\prime}$ is another orientable $n$-manifold with $\partial W^{\prime}=P^{\prime} \cup Q^{\prime}$, and $f:(W, \partial W) \rightarrow$ $\left(W^{\prime}, \partial W^{\prime}\right)$ is a map with $f(P) \subset P^{\prime}$ and $f(Q) \subset Q^{\prime}$, then the naturality of the cap product gives a commuting diagram:

$$
\begin{array}{ccc}
H^{k}(W, P ; \mathbb{Z}) & \stackrel{\cap[W]}{\longrightarrow} & H_{n-k}(W, Q ; \mathbb{Z}) . \\
f^{*} \uparrow & & \downarrow f_{*} \\
H^{k}\left(W^{\prime}, P^{\prime} ; \mathbb{Z}\right) & \stackrel{\cap f_{*}[W]}{\longrightarrow} & H_{n-k}\left(W^{\prime}, Q^{\prime} ; \mathbb{Z}\right)
\end{array}
$$

If $f$ is of degree \pm 1 , then both horizontal homomorphisms are isomorphisms, and hence $f_{*}$ is surjective.

For non-orientable manifolds, one may obtain duality isomorphisms and a diagram like $(\ddagger)$ by using $\mathbb{Z}_{2}$-coefficients. A more powerful duality theorem and 
corresponding version of $(\ddagger)$ for non-orientable manifolds may be obtained by using "twisted integer" coefficients. This will be discussed after we handle the orientable case.

Proof of Proposition 3.3 (orientable case with $\mathbb{Z}$-coefficients) Let $M^{n}$ be orientable and let $\left\{N_{i}\right\}_{i=0}^{\infty}$ be a sequence of neighborhoods of infinity along with the embeddings, rays, base points, subspaces and homotopies $\left\{K^{i}\right\}_{i=0}^{\infty}$ described earlier. For each $j \geq 0, H_{j}\left(\varepsilon\left(M^{n}\right) ; \mathbb{Z}\right)$ is represented by

$$
H_{j}\left(N_{0} ; \mathbb{Z}\right) \stackrel{\lambda_{1}}{\longleftarrow} H_{j}\left(N_{1} ; \mathbb{Z}\right) \stackrel{\lambda_{2}}{\longleftarrow} H_{j}\left(N_{2} ; \mathbb{Z}\right) \stackrel{\lambda_{3}}{\longleftarrow} \cdots
$$

where all bonding maps are induced by inclusion.

Since each $N_{i}$ is connected, $H_{0}\left(\varepsilon\left(M^{n}\right) ; \mathbb{Z}\right)$ is pro-equivalent to

$$
\mathbb{Z} \cong \mathbb{Z} \cong \mathbb{Z} \cong \ldots
$$

and thus, is stable. Let $j \geq 1$ be fixed.

Claim $1 \quad H_{j}\left(\varepsilon\left(M^{n}\right) ; \mathbb{Z}\right)$ is semistable.

We will show that, for each $[\alpha] \in H_{j}\left(N_{i+1}\right)$, there is a $\left[\alpha^{\prime}\right] \in H_{j}\left(N_{i+2}\right)$ such that $\alpha$ is homologous to $\alpha^{\prime}$ in $N_{i}$. Thus, $i m\left(\lambda_{i+1}\right) \stackrel{\lambda_{i+1}}{\longleftarrow} i m\left(\lambda_{i+2}\right)$ is surjective.

We may assume that $\alpha$ is supported in $A_{i+1}$. We abuse notation slightly and write $[\alpha] \in H_{j}\left(A_{i+1} ; \mathbb{Z}\right)$. Let $L^{i}: \partial N_{i+2} \times[0,1] \rightarrow N_{i}$ be the restriction of $K^{i}$. Note that $L^{i}\left(\partial N_{i+2} \times\{1\}\right) \subset A_{i}-\partial N_{i+1}$. By PL transversality theory (see [13] or Section II.4 of [1]), we may - after a small adjustment that does not alter $L^{i}$ on $\left(\partial N_{i+2} \times\{0,1\}\right) \cup\left(D_{i} \times[0,1]\right)$-assume that that $C_{i+1} \equiv\left(L^{i}\right)^{-1}\left(A_{i+1}\right)$ is an $n$-manifold with boundary ${ }^{1}$. Let $C_{i+1}^{*}$ be the component of $C_{i+1}$ that contains $D_{i}^{\prime} \times\left[0, \frac{1}{4}\right]$. Then $L^{i}$ takes $\partial C_{i+1}^{*}$ into $\partial A_{i+1}$ and, provided our adjustment to $L^{i}$ was sufficiently small, $L^{i}$ is still a homeomorphism over $T_{0}^{\prime} \cap A_{i+1}$. By the local characterization of degree, $\left.L^{i}\right|_{C_{i+1}^{*}}:\left(C_{i+1}^{*}, \partial C_{i+1}^{*}\right) \rightarrow\left(A_{i+1}, \partial A_{i+1}\right)$ is a degree 1 map. Thus, by an application of $(\ddagger),[\alpha]$ has a preimage $[\beta] \in$ $H_{j}\left(C_{i+1}^{*} ; \mathbb{Z}\right)$. Now $C_{i+1}^{*} \subset \partial N_{i+2} \times[0,1]$, and within the larger space, $\beta$ is homologous to a cycle $\beta^{\prime}$ supported in $\partial N_{i+2} \times\{0\}$. Since $L^{i}$ takes $\partial N_{i+2} \times[0,1]$ into $N_{i}$, it follows that $\alpha$ is homologous to $\alpha^{\prime} \equiv L^{i}\left(\beta^{\prime}\right) \subset \partial N_{i+2}$ in $N_{i}$.

\footnotetext{
${ }^{1}$ Instead of using transversality theory, we could simply use the radial structure of regular neighborhoods to alter $L^{i}$ in a thin regular neighborhood of $\left(L^{i}\right)^{-1}\left(A_{i} \cup N_{i+2}\right)$. Using this approach, we "fatten" the preimage of $A_{i} \cup N_{i+2}$ to a codimension 0 submanifold, thus ensuring that $\left(L^{i}\right)^{-1}\left(A_{i+1}\right)$ is an $n$-manifold with boundary.
} 
Claim $2 H_{j}\left(\varepsilon\left(M^{n}\right) ; \mathbb{Z}\right)$ is pro-monomorphic.

We'll show that $i m\left(\lambda_{i+2}\right) \stackrel{\lambda_{i+1}}{\longleftarrow} i m\left(\lambda_{i+3}\right)$ is injective, for all $i \geq 0$. It suffices to show that each $j$-cycle $\alpha$ in $N_{i+3}$ that bounds a $(j+1)$-chain $\gamma$ in $N_{i+1}$, bounds a $(j+1)$-chain in $N_{i+2}$. Let $\left[\gamma^{\prime}\right]$ be a preimage of $[\gamma]$ under the excision isomorphism

$$
H_{j+1}\left(A_{i+1} \cup A_{i+2}, \partial N_{i+3} ; \mathbb{Z}\right) \rightarrow H_{j+1}\left(N_{i+1}, N_{i+3} ; \mathbb{Z}\right) .
$$

Then $\alpha^{\prime} \equiv \partial \gamma^{\prime}$ is homologous to $\alpha$ in $N_{i+3}$, so it suffices to show that $\alpha^{\prime}$ bounds in $N_{i+2}$.

By passing to a subsequence if necessary, we may assume that the image of $\partial N_{i+2} \times[0,1]$ under $K^{i}$ lies in $A_{i} \cup A_{i+1} \cup A_{i+2}-U$, where $U$ is a collar neighborhood of $\partial N_{i+3}$ in $A_{i+2}$. Then define

$$
f:\left(\partial N_{i+2} \times[0,1]\right) \cup A_{i+2} \rightarrow A_{i} \cup A_{i+1} \cup A_{i+2}
$$

to be $K^{i}$ on $\partial N_{i+2} \times[0,1]$ and the identity on $A_{i+2}$. Arguing as in the proof of Claim 1, we may - without changing the map on $A_{i+2}$-make a small adjustment to $f$ so that $C \equiv f^{-1}\left(A_{i+1} \cup A_{i+2}\right)$ is an $n$-manifold with boundary. Let $C^{*}$ be the component that contains $A_{i+2}$. Then $f$ takes $\partial N_{i+3}$ onto $\partial N_{i+3}$, and $P \equiv \partial C^{*}-\partial N_{i+3}$ to $\partial N_{i+1}$. Provided our adjustment was sufficiently small, $f$ is a homeomorphism over $U$, so $f:\left(C^{*}, \partial C^{*}\right) \rightarrow\left(A_{i+1} \cup A_{i+2}, \partial N_{i+1} \cup \partial N_{i+3}\right)$ is a degree 1 map Applying $(\ddagger)$ to this situation we obtain a surjection

$$
H_{j+1}\left(C, \partial N_{i+3} ; \mathbb{Z}\right) \rightarrow H_{j+1}\left(A_{i+1} \cup A_{i+2}, \partial N_{i+3} ; \mathbb{Z}\right) .
$$

Let $[\eta]$ be a preimage of $\left[\alpha^{\prime}\right]$. Utilizing the product structure on $\partial N_{i+2} \times[0,1]$, we may retract $C^{*}$ onto $A_{i+2}$. The image $\eta^{\prime}$ of $\eta$ under this retraction is a relative $(j+1)$-cycle in $\left(A_{i+2}, \partial N_{i+3}\right)$ with $\partial \eta^{\prime}=\partial \eta$. Thus, $\partial \eta^{\prime}$ is homologous to $\partial \gamma^{\prime}=\alpha^{\prime}$, so $\alpha^{\prime}$ bounds in $A_{i+2} \subset N_{i+2}$ as desired.

Before proceeding with the proof of the non-orientable case, we discuss some necessary background. The proof just presented already works for non-orientable manifolds if we replace the coefficient ring $\mathbb{Z}$ with $\mathbb{Z}_{2}$. To obtain the result for $\mathbb{Z}$-coefficients (and ultimately an arbitrary coefficient ring), we will utilize homology with twisted integer coefficients, which we will denote by $\widetilde{\mathbb{Z}}$. The key here is that, even for a non-orientable compact $n$-manifold with boundary, $H_{n}(W, \partial W ; \widetilde{\mathbb{Z}}) \cong \mathbb{Z}$. Thus, we have an orientation class $[W]$ and it may be used to obtain a duality isomorphism - where homology is now taken with twisted integer coefficients. Furthermore, if a map $f:(W, \partial W) \rightarrow\left(W^{\prime}, \partial W^{\prime}\right)$ is 
orientation true (meaning that $f$ takes orientation reversing loops to orientation reversing loops and orientation preserving loops to orientation preserving loops), then we have a well defined notion of $\operatorname{deg}(f) \in \mathbb{Z}$. These versions of duality and degree yield an analogous version of diagram ( $\ddagger)$, which tells us that degree \pm 1 maps (appropriately defined) between compact (possibly non-orientable) manifolds with boundary induce surjections on homology with $\widetilde{\mathbb{Z}}$-coefficients. See section 3.H of [10] or Chapter 2 of [18] discussions of homology with coefficients in $\widetilde{\mathbb{Z}}$, and [12] for a discussion of degree of a map between non-orientable manifolds.

As with the traditional definition of degree, this generalized version can be detected locally. In particular, an orientation true map $f:(W, \partial W) \rightarrow\left(W^{\prime}, \partial W^{\prime}\right)$ that is a homeomorphism over some open subset of $W^{\prime}$ has degree \pm 1 . See [12, $3.8]$.

For non-orientable $W$, let $p: \widehat{W} \rightarrow W$ be the orientable double covering projection. Then there is a long exact sequence:

$$
\cdots \rightarrow H_{k}(W ; \widetilde{\mathbb{Z}}) \rightarrow H_{k}(\widehat{W} ; \mathbb{Z}) \stackrel{p_{*}}{\rightarrow} H_{k}(W ; \mathbb{Z}) \rightarrow H_{k-1}(W ; \widetilde{\mathbb{Z}}) \rightarrow \cdots
$$

This sequence is natural with respect to orientation true mappings $f: W \rightarrow W^{\prime}$. See section $3 . \mathrm{H}$ of [10] for a discussion of this sequence.

\section{Proof of Proposition 3.3 (non-orientable case with $\mathbb{Z}$-coefficients)}

Let $M^{n}$ be one ended, inward tame, and have compact boundary. If $M^{n}$ contains an orientable neighborhood of infinity, we can simply disregard its complement and apply the orientable case. Hence, we assume that $\left\{N_{i}\right\}_{i=0}^{\infty}$ is a nested cofinal sequence of 0 -neighborhoods of infinity, each of which is non-orientable.

The first step in this proof is to observe that, if we use homology with $\widetilde{\mathbb{Z}}$ coefficients, the proof used in the orientable case is still valid. A few points are worth noting. First, the inclusion maps $N_{i} \hookrightarrow N_{i+1}$ are clearly orientation true. Similarly, since each $\partial N_{i}$ is bicollared in $M^{n}$, orientation reversing [preserving] loops in $\partial N_{i}$ are orientation reversing [preserving] in $M^{n}$. Hence, the maps $L^{i}: \partial N_{i+2} \times[0,1] \rightarrow N_{i}$ (and restrictions to codimension 0 submanifolds) are also orientation true. With this, and the additional ingredients discussed above, we see that $H_{j}\left(\varepsilon\left(M^{n}\right) ; \widetilde{\mathbb{Z}}\right)$ is stable for all $j$.

The second step is to consider the orientable double covering projection $p$ : $\widehat{M}^{n} \rightarrow M^{n}$. For each $i, \widehat{N}_{i}=p^{-1}\left(N_{i}\right)$ is the orientable double cover of $N_{i}$, and thus, is connected. It follows that $\widehat{M}^{n}$ is one ended, with $\left\{\widehat{N}_{i}\right\}_{i=0}^{\infty}$ a cofinal 
sequence of 0-neighborhoods of infinity. Furthermore, taming homotopies for $M^{n}$ may be lifted to obtain taming homotopies for $\widehat{M}^{n}$, so $\widehat{M}^{n}$ is inward tame. It follows from the orientable case that $H_{j}\left(\varepsilon\left(\widehat{M}^{n}\right) ; \mathbb{Z}\right)$ is stable for all $j$.

Next we apply the long exact discussed above to each covering projection $p_{i}$ : $\widehat{N_{i}} \rightarrow N$. Together with naturality, this yields a long exact sequence of inverse sequences:

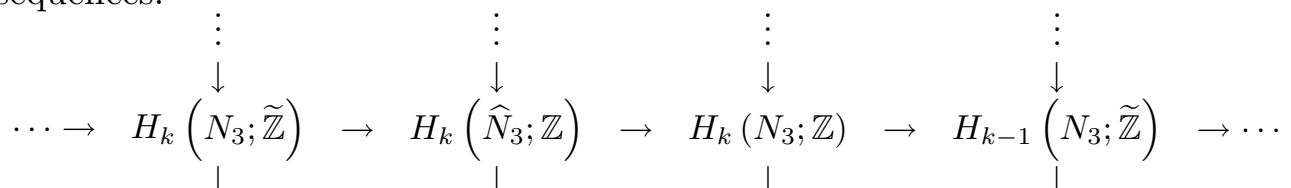

$$
\begin{aligned}
& \cdots \rightarrow \quad H_{k}\left(N_{2} ; \widetilde{\mathbb{Z}}\right) \rightarrow H_{k}\left(\begin{array}{c}
\left.\widehat{N}_{2} ; \mathbb{Z}\right) \\
\downarrow
\end{array} \rightarrow \quad H_{k}\left(N_{2} ; \mathbb{Z}\right) \rightarrow H_{k-1}\left(N_{2} ; \widetilde{\mathbb{Z}}\right) \rightarrow \cdots\right. \\
& \cdots \rightarrow H_{k}\left(N_{1} ; \widetilde{\mathbb{Z}}\right) \rightarrow H_{k}\left(\widehat{N}_{1} ; \mathbb{Z}\right) \rightarrow H_{k}\left(N_{1} ; \mathbb{Z}\right) \rightarrow H_{k-1}\left(N_{1} ; \widetilde{\mathbb{Z}}\right) \rightarrow \cdots
\end{aligned}
$$

We may now apply Lemma 2.3 to conclude that $H_{j}\left(\varepsilon\left(M^{n}\right) ; \mathbb{Z}\right)$ is stable for all $j$.

Lastly, we generalize the above to the case of an arbitrary coefficient ring.

Proof of Proposition 3.3 ( $R$-coefficients) Now let $R$ be ring with unity. By applying the Universal Coefficient Theorem for homology (see [10, Cor. 3.A.4]) to obtain each row, we may get (for each $j$ ) the following diagram:

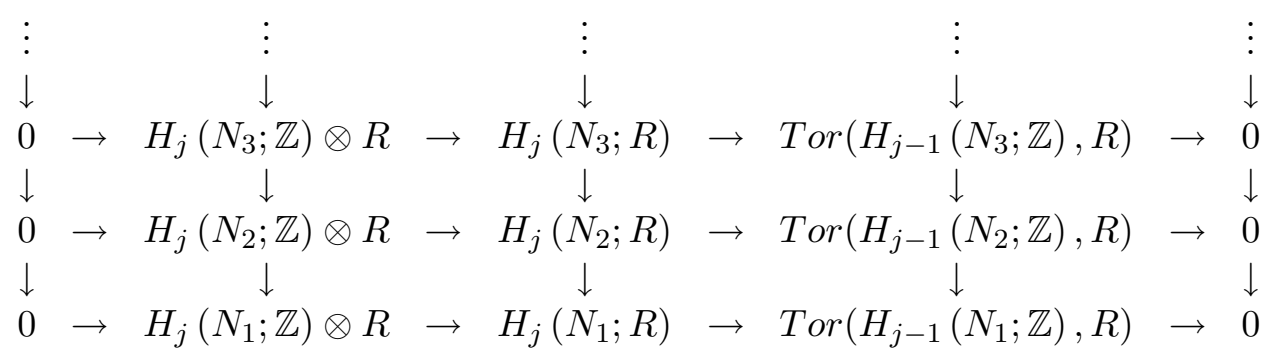

The second and fourth columns are stable by the $\mathbb{Z}$-coefficient case, so an application of Lemma 2.3 yields stability of $H_{j}\left(\varepsilon\left(M^{n}\right) ; R\right)$.

Remark 5 A variation on the above can be used to show that, for one ended manifolds with compact boundary, inward tameness plus $\pi_{1}$-stability implies $\pi_{2}$-stability. To do this, begin with a cofinal sequence $\left\{N_{i}\right\}$ of (strong) 1neighborhoods of infinity - see Theorem 4 of [7]. Then show that the inverse sequence

$$
H_{2}\left(\widetilde{N}_{0} ; \mathbb{Z}\right) \leftarrow H_{2}\left(\widetilde{N}_{1} ; \mathbb{Z}\right) \leftarrow H_{2}\left(\widetilde{N}_{2} ; \mathbb{Z}\right) \leftarrow \cdots
$$


is stable, where each $\widetilde{N}_{i}$ is the universal cover of $N_{i}$. This will require Poincaré duality for noncompact manifolds; otherwise, the proof simply mimics the proof of Prop. 3.3. It follows from the Hurewicz theorem that

$$
\pi_{2}\left(\tilde{N}_{0}, \tilde{p}_{0}\right) \leftarrow \pi_{2}\left(\tilde{N}_{1}, \tilde{p}_{1}\right) \leftarrow \pi_{2}\left(\tilde{N}_{2}, \tilde{p}_{2}\right) \leftarrow \cdots
$$

is stable, and hence, so is $\pi_{2}\left(\varepsilon\left(M^{n}\right)\right)$. As an application of this observation, one may deduce the main result of Siebenmann's thesis as a direct corollary of Theorem 1.1-provided $n \geq 7$.

\section{Proof of Theorem 1.3}

In this section we will construct (for each $n \geq 6$ ) a one ended open $n$-manifold $M_{*}^{n}$ in which all clean neighborhoods of infinity have finite homotopy type, yet $\pi_{1}\left(\varepsilon\left(M_{*}^{n}\right)\right)$ is not perfectly semistable. Hence $M_{*}^{n}$ satisfies conditions (1) and (3) of Theorem 1.1, but is not pseudo-collarable.

In the first portion of this section we present the necessary group theory on which the examples rely. In the next portion, we give a detailed construction of the examples and verify the desired properties.

\subsection{Group Theory}

We assume the reader is familiar with the basic notions of group presentations in terms of generators and relators. We use the HNN-extension as our basic building block. A more thorough discussion of HNN-extensions may be found in [11] or [4].

Before beginning, we describe the algebraic goal of this section. We wish to construct a special inverse sequence of finitely presented groups that is semistable, but not perfectly semistable. Later this sequence will be realized as the fundamental group at infinity of a carefully constructed open manifold. The following lemma indicates the strategy that will be used.

\section{Lemma 4.1 Let}

$$
G_{0} \stackrel{\psi_{1}}{\longleftarrow} G_{1} \stackrel{\psi_{2}}{\longleftarrow} G_{2} \stackrel{\psi_{3}}{\longleftarrow} G_{3} \stackrel{\psi_{4}}{\longleftarrow} \ldots
$$

be an inverse sequence of groups with surjective but non-injective bonding homomorphisms. Suppose further that no $G_{i}$ contains a non-trivial perfect subgroup. Then this inverse sequence is not perfectly semistable. 
Proof It is easy to see that this system is semistable but not stable. Assume that it is perfectly semistable. Then-after passing to a subsequence, relabelling, and applying Lemma 2.1 - we may assume the existence of a diagram:

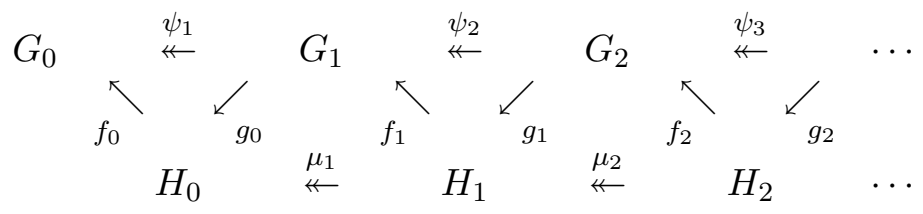

where each $\mu_{i}$ has a perfect kernel.

By the commutativity of the diagram, all of the $f_{i}$ 's and $g_{i}$ 's are surjections. Moreover, Lemma 2.2 implies that $f_{i}\left(\operatorname{ker} \mu_{i}\right)=\{1\}$, for all $i \geq 1$. The combination of these facts tells us that each $g_{i}$ is an isomorphism. Since the $G_{i}$ 's contain no nontrivial perfect subgroups, then neither do the $H_{i}$ 's. But then each $\mu_{i}$ is an isomorphism, contradicting the non-stability of our original sequence.

Remark 6 Satisfying the hypotheses of Lemma 4.1 by itself is not difficult. For example, since abelian groups contain no nontrivial perfect subgroups, examples such as

$$
\mathbb{Z} \nleftarrow \mathbb{Z} \oplus \mathbb{Z} \nleftarrow \mathbb{Z} \oplus \mathbb{Z} \oplus \mathbb{Z} \nleftarrow \cdots
$$

apply. However, Theorem 1.2 tells us that this inverse sequence cannot occur as the fundamental group at infinity of an inward tame open manifold. Indeed, any appropriate inverse sequence should, at least, have the property that abelianizing each term yields a stable sequence. Thus, our task of constructing an appropriate "realizable" inverse sequence is rather delicate.

Let $K$ be a group with presentation $\langle\operatorname{gen}(K) \mid \operatorname{rel}(K)\rangle$ and $\left\{\phi_{i}\right\}$ a collection of monomorphisms $\phi_{i}: L_{i} \rightarrow K$ from subgroups $\left\{L_{i}\right\}$ of $K$ into $K$. We define the group

$$
G=\left\langle\operatorname{gen}(K), t_{1}, t_{2}, \cdots \mid \operatorname{rel}(K), R_{1}, R_{2}, \cdots\right\rangle
$$

where each $R_{i}$ is the collection of relations $\left\{t_{i} l_{i j} t_{i}^{-1}=\phi_{i}\left(l_{i j}\right)\right.$ for all $\left.l_{i j} \in L_{i}\right\}$. We call $G$ the $H N N$ group with base $K$, associated subgroups $\left\{L_{i}, \phi_{i}\left(L_{i}\right)\right\}$, and free part the group generated by $\left\{t_{1}, t_{2}, \cdots\right\}$. We assume the basic properties of HNN groups - such as the fact that the base group naturally embeds in the HNN group. This and other basic structure theorems for subgroups of HNNextensions have existed for a long time and appear within many sources. Most important for our purposes is the following which we have tailored to meet our specific needs. 
Theorem 4.2 (see [11, Theorem 6]) Let $G$ be the HNN group above. If $H$ is a subgroup of $G$ having trivial intersection with the conjugates of each $L_{i}$, then $H$ is the free product of a free group with the intersections of $H$ with certain conjugates of $K$.

Let $a$ and $b$ be group elements. We denote by $[a, b]$ the commutator of $a$ and $b$, ie, $[a, b]=a^{-1} b^{-1} a b$. Let $S$ be a subset of elements of a group $G$. We denote by $\left\{s_{1}, s_{2}, \cdots ; G\right\}$ the subgroup of $G$ generated by $S$ where $S=\left\{s_{1}, s_{2}, \cdots\right\}$. If $S$ and $G$ are as above, then we denote by ncl $\left\{s_{1}, s_{2}, \cdots ; G\right\}$ the normal closure of $S$ in $G$, ie, the smallest normal subgroup of $G$ containing $S$.

We are now ready to construct the desired inverse sequence. Let $G_{0}=\left\langle a_{0}\right\rangle$ be the free group on one generator. Of course, $G_{0}$ is just $\mathbb{Z}$ written multiplicatively. For $j \geq 1$, let

$$
G_{j}=\left\langle a_{0}, a_{1}, \cdots, a_{j} \mid a_{1}=\left[a_{1}, a_{0}\right], a_{2}=\left[a_{2}, a_{1}\right], \cdots, a_{j}=\left[a_{j}, a_{j-1}\right]\right\rangle
$$

This presentation emphasizes that each $a_{i}(i \geq 1)$ is a commutator. (Hence, each $G_{j}$ abelianizes to $\mathbb{Z}$.) We abuse notation slightly and do not distinguish between the element $a_{i} \in G_{j-1}$ and $a_{i} \in G_{j}$. Let $j \geq 1$; another useful presentation of $G_{j}$ is

$$
G_{j}=\left\langle a_{0}, a_{1}, \cdots, a_{j} \mid a_{0} a_{1}^{2} a_{0}^{-1}=a_{1}, a_{1} a_{2}^{2} a_{1}^{-1}=a_{2}, \cdots, a_{j-1} a_{j}^{2} a_{j-1}^{-1}=a_{j}\right\rangle
$$

Now, $G_{j}$ can be put in the form of an HNN group. In particular,

$$
G_{j}=\left\langle\operatorname{gen}(K), t_{1} \mid \operatorname{rel}(K), R_{1}\right\rangle
$$

where

$$
K=\left\langle a_{1}, a_{2}, \cdots, a_{j} \mid a_{1} a_{2}^{2} a_{1}^{-1}=a_{2}, a_{2} a_{3}^{2} a_{2}^{-1}=a_{3}, \cdots, a_{j-1} a_{j}^{2} a_{j-1}^{-1}=a_{j}\right\rangle,
$$

$t_{1}=a_{0}, L_{1}=\left\{a_{1}^{2} ; G_{j}\right\}, \phi_{1}\left(a_{1}^{2}\right)=a_{1}$, and $R_{1}$ is given by $a_{0} a_{1}^{2} a_{0}^{-1}=a_{1}$. The base group, $K$, is obviously isomorphic to $G_{j-1}$ with that isomorphism taking $a_{i}$ to $a_{i-1}$.

Define $\psi_{j}: G_{j} \rightarrow G_{j-1}$ by sending $a_{i}$ to $a_{i}$ for $1 \leq i \leq j-1$, and $a_{j}$ to 1 . By inspection $\psi_{j}$ is a surjective homomorphism. Our goal is to prove:

Theorem 4.3 In the setting described above, the group $G_{j}$ has no non-trivial perfect subgroups.

Proof Our proof is by induction. 
Case $j=0 \quad G_{0}=\left\langle a_{0}\right\rangle$ is an abelian group so that all commutators in $G_{0}$ are trivial. Thus, $[H, H]=1$ for any subgroup $H$ of $G_{0}$. Hence, $H=1$ is the only perfect subgroup of $G_{0}$.

Case $j=1 \quad$ Consider $G_{1}$ and $\psi_{1}: G_{1} \rightarrow G_{0} . \psi_{1}:\left\langle a_{0}, a_{1} \mid a_{0} a_{1}^{2} a_{0}^{-1}=a_{1}\right\rangle \rightarrow$ $\left\langle a_{0}\right\rangle$. We pause to observe for later use that $G_{1}$ is an HNN group with base group $K=\left\{a_{1} ; G_{1}\right\}$. Since $K$ embeds in $G_{1}$, then $a_{1}$ has infinite order in $G_{1}$. Now, $G_{1}$ is one of the well-known Baumslag-Solitar groups. Its commutator subgroup, $\left[G_{1}, G_{1}\right]$, is precisely equal to $\operatorname{ker}\left(\psi_{1}\right)$. The substitution $b_{k}=a_{0}^{-k} a_{1} a_{0}^{k}$ along with the relations

$$
b_{k}=a_{0}^{-k} a_{1} a_{0}^{k}=a_{0}^{-(k-1)}\left(a_{0}^{-1} a_{1} a_{0}^{1}\right) a_{0}^{k-1}=a^{-(k-1)} a_{1}^{2} a^{k-1}=\left(a^{-(k-1)} a_{1} a^{k-1}\right)^{2}
$$

give $\operatorname{ker}\left(\psi_{1}\right)$ a presentation:

$$
\left\langle b_{k} \mid b_{k}=b_{k-1}^{2},-\infty<j<\infty\right\rangle
$$

So, $\operatorname{ker}\left(\psi_{1}\right)$ is locally cyclic (every finitely generated subgroup is contained in a cyclic subgroup). In particular, it is abelian and contains no non-trivial perfect subgroups. Now, suppose $P$ is a perfect subgroup of $G_{1}$. Then, by Lemma $2.2, \psi_{1}(P)$ is a perfect subgroup of $G_{0}$. By the case $(j=0), \psi_{1}(P)=\{1\}$, so $P \subset \operatorname{ker}\left(\psi_{1}\right)$. But, we just observed, then, that $P$ must be trivial.

Inductive Step We assume that $G_{j}$ contains no non-trivial perfect subgroups for $1 \leq j \leq k-1$ and prove that $G_{k}$ has this same property. To this end, let $P$ be a perfect subgroup of $G_{k}$. Then, $\psi_{k}(P)$ is a perfect subgroup of $G_{k-1}$. By induction, $\psi_{k}(P)=1$. Thus, $P \subset \operatorname{ker}\left(\psi_{k}\right)$.

As shown above, $G_{k}$, is an HNN-extension with base group $K$ where

$$
\begin{aligned}
K & =\left\langle a_{1}, a_{2}, \cdots, a_{k} \mid a_{1} a_{2}^{2} a_{1}^{-1}=a_{2}, a_{2} a_{3}^{2} a_{2}^{-1}=a_{3}, \cdots, a_{k-1} a_{k}^{2} a_{k-1}^{-1}=a_{k}\right\rangle \\
& \cong G_{k-1}
\end{aligned}
$$

By the inductive hypothesis, $K$ has no perfect subgroups. Moreover, $a_{1} \in K$ still has infinite order in both $K$ (by induction) and $G_{k}$ (since $K$ embeds in $\left.G_{k}\right)$. Moreover, the HNN group, $G_{k}$, has the single associated cyclic subgroup, $L=\left\{a_{1}^{2} ; G_{k}\right\}$, with conjugation relation $a_{0} a_{1}^{2} a_{0}^{-1}=a_{1}$. By the definition of $\psi_{k}: G_{k} \rightarrow G_{k-1}$ it is clear that $\operatorname{ker}\left(\psi_{k}\right)=\operatorname{ncl}\left\{a_{k} ; G_{k}\right\}$.

Claim No conjugate of $L$ non-trivially intersects $n c l\left\{a_{k} ; G_{k}\right\}$

Proof of Claim If the claim is false, then $L$ itself must non-trivially intersect the normal subgroup, $n c l\left\{a_{k} ; G_{k}\right\}$. This means that $a_{1}^{2 m} \in \operatorname{ncl}\left\{a_{k} ; G_{k}\right\}=$ 
$\operatorname{ker}\left(\psi_{k}\right)$ for some integer $m>0$. Since $k \geq 2$, then $\psi_{k}\left(a_{1}^{2 m}\right)=\psi_{k}\left(a_{1}\right)^{2 m}=$ $a_{1}^{2 m}=1$ in $G_{k-1}$, ie, $a_{1}$ has finite order in $G_{k-1}$. This contradicts our observations above, thus proving the claim.

We continue with the proof of Theorem 4.3. Recall that $P$ is a perfect subgroup of $\operatorname{ker}\left(\psi_{k}\right)$. It must also enjoy the property of trivial intersection with each conjugate of $L$. We now apply Theorem 4.2 to the subgroup $P$ to conclude that $P$ is a free product where each factor is either free or equal to $P \cap g K g^{-1}$ for some $g \in G_{k}$.

Now, $P$ projects naturally onto each of these factors so each factor is perfect. However, non-trivial free groups are not perfect. Moreover, by induction, $K$ (or equivalently $g K^{-1}$ ) contains no non-trivial perfect subgroups. Thus, any subgroup, $P \cap g K g^{-1}$, is trivial. Consequently, $P$ must be trivial.

\subsection{Construction of $M_{*}^{n}$}

The goal of this section is to construct a one ended open $n$-manifold $M_{*}^{n}(n \geq 6)$ with fundamental group system at infinity equivalent to the inverse sequence

$$
G_{0} \stackrel{\psi_{1}}{\longleftarrow} G_{1} \stackrel{\psi_{2}}{\longleftarrow} G_{2} \stackrel{\psi_{3}}{\longleftarrow} G_{3} \stackrel{\psi_{4}}{\longleftarrow} \cdots
$$

produced above. More importantly, this will be done in such a way that clean neighborhoods of infinity in $M_{*}^{n}$ have finite homotopy type - thereby proving Theorem 1.3. Familiarity with the basics of handle theory, as can be found in Chapter 6 of [14], is assumed throughout the construction.

The key to producing $M_{*}^{n}$ will be a careful construction of a sequence

$$
\left\{\left(A_{i}, \Gamma_{i}, \Gamma_{i+1}\right)\right\}_{i=0}^{\infty}
$$

of compact $n$-dimensional cobordisms satisfying the following properties:

a) The left-hand boundary $\Gamma_{0}$ of $A_{0}$ is $S^{n-2} \times S^{1}$, and (as indicated by the notation), for all $i \geq 1$ the left-hand boundary of $A_{i}$ is equal to the right-hand boundary of $A_{i-1}$. In particular, $A_{i-1} \cap A_{i}=\Gamma_{i}$.

b) For all $i \geq 0, \pi_{1}\left(\Gamma_{i}, p_{i}\right) \cong G_{i}$ and $\Gamma_{i} \hookrightarrow A_{i}$ induces a $\pi_{1}$-isomorphism.

c) The isomorphisms between $\pi_{1}\left(\Gamma_{i}, p_{i}\right)$ and $G_{i}$ may be chosen so that we have a commutative diagram:

$$
\begin{array}{ccc}
G_{i} & \stackrel{\psi_{i+1}}{\longleftarrow} & G_{i+1} \\
\downarrow & & \downarrow \cong \\
\pi_{1}\left(\Gamma_{i}, p_{i}\right) & \stackrel{\mu_{i+1}}{\longleftarrow} & \pi_{1}\left(\Gamma_{i+1}, p_{i+1}\right)
\end{array}
$$

Geometry $8 \mathcal{T}$ Topology, Volume 7 (2003) 
Here $\mu_{i+1}$ is the composition of homomorphisms

$$
\pi_{1}\left(\Gamma_{i}, p_{i}\right) \stackrel{\cong}{\leftrightarrows} \pi_{1}\left(A_{i}, p_{i}\right) \stackrel{\widehat{\alpha}_{i}}{\longleftarrow} \pi_{1}\left(A_{i}, p_{i+1}\right) \stackrel{j_{\#}}{\longleftarrow} \pi_{1}\left(\Gamma_{i+1}, p_{i+1}\right)
$$

where $j_{\#}$ is induced by inclusion, the middle map is a "change of base points isomorphism" with respect to a path $\alpha_{i}$ in $A_{i}$ between $p_{i}$ and $p_{i+1}$, and the left-most isomorphism is provided by property $\mathrm{b}$ ).

We will let

$$
M_{*}^{n}=\left(S^{n-2} \times B^{2}\right) \cup A_{0} \cup A_{1} \cup A_{2} \cup \cdots
$$

where $S^{n-2} \times B^{2}$ is glued to $A_{0}$ along $\Gamma_{0}=S^{n-2} \times S^{1}$. Then for each $i \geq 0$,

$$
N_{i}=A_{i} \cup A_{i+1} \cup A_{i+2} \cup \cdots
$$

is a clean connected neighborhood of infinity. Moreover, by properties b) and c) and repeated application of the Seifert-VanKampen theorem, the inverse sequence

$$
\pi_{1}\left(N_{0}, p_{0}\right) \stackrel{\lambda_{1}}{\longleftarrow} \pi_{1}\left(N_{1}, p_{1}\right) \stackrel{\lambda_{2}}{\longleftarrow} \pi_{1}\left(N_{2}, p_{2}\right) \stackrel{\lambda_{3}}{\longleftarrow} \cdots
$$

is isomorphic to $(\dagger \dagger)$.

Finally, we will need to show that clean neighborhoods of infinity in $M_{*}^{n}$ have finite homotopy type. This can be done only after the specifics of the construction are revealed.

Step 0 Construction of $\left(A_{0}, \Gamma_{0}, \Gamma_{1}\right)$.

Let $\Gamma_{0}=S^{n-2} \times S^{1}$ and $p_{0} \in \Gamma_{0}$. Keeping in mind that $G_{0}=\left\langle a_{0}\right\rangle$, we abuse notation slightly by letting $a_{0}$ also represent a generator of $\pi_{1}\left(\Gamma_{0}, p_{0}\right) \cong \mathbb{Z}$. This gives a canonical isomorphism from $G_{0}$ to $\pi_{1}\left(\Gamma_{0}, p_{0}\right)$.

Let $\varepsilon$ be a small positive number and $C_{0}^{\prime}=\Gamma_{0} \times[1-\varepsilon, 1]$. To the left-hand boundary component of $C_{0}^{\prime}$ attach an orientable 1-handle $h_{0}^{1}$. Note that $C_{0}^{\prime} \cup h_{0}^{1}$ and its left boundary component each have fundamental group that is free on two generators-the first corresponding to $a_{0}$, and the second corresponding to a circle that runs once through $h_{0}^{1}$. Denote this second generator by $a_{1}$. Keeping in mind the presentation $G_{1}=\left\langle a_{0}, a_{1} \mid a_{1}=\left[a_{1}, a_{0}\right]\right\rangle$, attach to the left-hand boundary component of $C_{0}^{\prime} \cup h_{0}^{1}$ a 2-handle $h_{0}^{2}$ along a regular neighborhood of a loop corresponding to $a_{1}^{-2} a_{0}^{-1} a_{1} a_{0}$. Let $B_{0}=C_{0}^{\prime} \cup h_{0}^{1} \cup h_{0}^{2}$ and let $\Gamma_{1}$ denote the left-hand boundary component of $B_{0}$. By avoiding the arc $p_{0} \times[1-\varepsilon, 1]$ when attaching $h_{0}^{1}$ and $h_{0}^{2}$, we may let $p_{1}=p_{0} \times\{1-\varepsilon\} \in \Gamma_{1}$. Clearly $\pi_{1}\left(B_{0}, p_{1}\right) \cong G_{1}$. By inverting the handle decomposition, we may view $B_{0}$ as the result of attaching an $(n-2)$-handle and then an $(n-1)$-handle to a small 
product neighborhood $C_{1}$ of $\Gamma_{1}$. Since these handles have index greater than $2, \Gamma_{1} \hookrightarrow B_{0}$ induces a $\pi_{1}$-isomorphism. Hence $\pi_{1}\left(\Gamma_{1}, p_{1}\right) \cong G_{1}$. This gives us a cobordism $\left(B_{0}, \Gamma_{1}, \Gamma_{0}\right)$ with the desired boundary components. However, it is not the cobordism we are seeking.

Next attach a 2-handle $k_{0}^{2}$ to $B_{0}$ along a circle in $\Gamma_{1}$ representing $a_{1}$. Note that $k_{0}^{2}$ and $h_{0}^{1}$ form a canceling handle pair in $C_{0}^{\prime} \cup h_{0}^{1} \cup h_{0}^{2} \cup k_{0}^{2}$. Moreover, since $a_{1}$ has been killed, $h_{0}^{2}$ is now attached along a trivial loop in the left-hand boundary of $C_{0}^{\prime} \cup h_{0}^{1} \cup k_{0}^{2} \approx C_{0}^{\prime}$. Provided that $h_{0}^{2}$ was attached with the appropriate framing (this can still be arranged if necessary), we may attach a 3 -handle $k_{0}^{3}$ to $C_{0}^{\prime} \cup h_{0}^{1} \cup h_{0}^{2} \cup k_{0}^{2}$ that cancels $h_{0}^{2}$. Therefore, $C_{0}^{\prime} \cup h_{0}^{1} \cup h_{0}^{2} \cup k_{0}^{2} \cup k_{0}^{3} \approx \Gamma_{0} \times[0,1]$. The desired cobordism $\left(A_{0}, \Gamma_{0}, \Gamma_{1}\right)$ will be the complement of $B_{0}$ in this product. More precisely, $A_{0}=C_{1} \cup k_{0}^{2} \cup k_{0}^{3}$ where $C_{1}$ is a small product neighborhood of $\Gamma_{1}$ in $B_{0}$. By avoiding $p_{1}$ when attaching $k_{2}$ and $k_{3}$ we may let $p_{0}$ be the left endpoint of the collar line of $C_{1}$ having right end point corresponding to $p_{1}$. A schematic diagram of this setup is given in Figure 3.

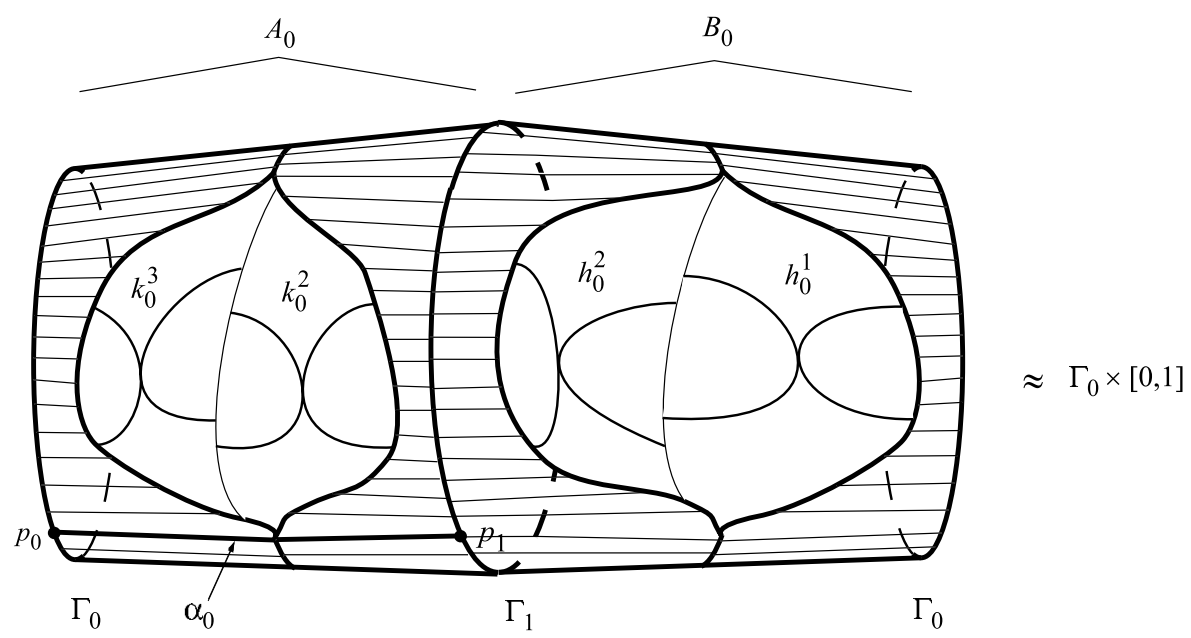

Figure 3

By Van Kampen's theorem, it is clear that $\pi_{1}\left(A_{0}, p_{1}\right) \cong\left\langle a_{0}\right\rangle$, and that the inclusion induced homomorphism $\pi_{1}\left(\Gamma_{1}, p_{1}\right) \rightarrow \pi_{1}\left(A_{0}, p_{1}\right)$ sends $a_{0}$ to $a_{0}$ and $a_{1}$ to 1 . By inverting the cobordism, we may view $A_{0}$ as the result of attaching an $(n-3)$ - and an $(n-2)$-handle to the right-hand boundary of $C_{0}=\Gamma_{0} \times$ $[0, \varepsilon]$. Hence, inclusion $\Gamma_{0} \hookrightarrow A_{0}$ induces the obvious $\pi_{1}$-isomorphism. It follows that properties a)-c) are satisfied by $\left(A_{0}, \Gamma_{0}, \Gamma_{1}\right)$.

Inductive Step Construction of $\left(A_{j}, \Gamma_{j}, \Gamma_{j+1}\right)$. 
Here we assume that $j \geq 1$ and that $\left(A_{j-1}, \Gamma_{j-1}, \Gamma_{j}\right)$ has already been constructed. We will construct $A_{j}$ from $\Gamma_{j}$ in the same manner that we constructed $A_{0}$ from $\Gamma_{0}$.

Given that $\pi_{1}\left(\Gamma_{j}, p_{j}\right) \cong G_{j}=\left\langle a_{0}, a_{1}, \cdots, a_{j}\right| a_{i}=\left[a_{i}, a_{i-1}\right]$ for all $\left.1 \leq i \leq j\right\rangle$, we expand the fundamental group by attaching a 1 -handle $h_{j}^{1}$ to the left-hand boundary component of $C_{j}^{\prime}=\Gamma_{j} \times[1-\varepsilon, 1]$. Let $a_{j+1}$ denote the fundamental group element of $C_{j}^{\prime} \cup h_{j}^{1}$ corresponding to a loop that runs once through $h_{j}^{1}$. Then attach to the left-hand boundary component of $C_{j}^{\prime} \cup h_{j}^{1}$ a 2-handle $h_{j}^{2}$ along a regular neighborhood of a loop corresponding to $a_{j+1}^{-2} a_{j}^{-1} a_{j+1} a_{j}$. This yields a cobordism $\left(B_{j}, \Gamma_{j+1}, \Gamma_{j}\right)$ with $\pi_{1}\left(B_{j}, p_{j+1}\right) \cong G_{j+1}$ and $\Gamma_{j+1} \hookrightarrow B_{j}$ inducing a $\pi_{1}$-isomorphism. Now attach a 2 -handle $k_{j}^{2}$ to $B_{j}$ along a circle in $\Gamma_{j+1}$ representing $a_{j+1}$. Reasoning as in the base case, we may then attach a 3-handle $k_{j}^{3}$ to cancel $h_{j}^{2}$ and giving

$$
C_{j}^{\prime} \cup h_{j}^{1} \cup h_{j}^{2} \cup k_{j}^{2} \cup k_{j}^{3} \approx \Gamma_{j} \times[0,1] .
$$

Let $C_{j+1}$ be a small product neighborhood of $\Gamma_{j+1}$ in $B_{j}$ and let

$$
A_{j}=C_{j+1} \cup k_{j}^{2} \cup k_{j}^{3} .
$$

Again, the same reasoning used in the base case shows that $\left(A_{j}, \Gamma_{j}, \Gamma_{j+1}\right)$ satisfies conditions a)-c).

Note In completing the proof of Theorem 1.3, we will utilize-in addition to properties a-c) - specific details and notation established in the above construction.

It remains to prove the following:

Proposition 4.4 Each clean neighborhood of infinity in $M_{*}^{n}$ has finite homotopy type.

Proof It suffices to find one cofinal sequence of clean neighborhoods of infinity with this property. For each $i \geq 1$, let $N_{i}^{\prime}=N_{i} \cup k_{i-1}^{2}$, where $N_{i}=A_{i} \cup A_{i+1} \cup$ $A_{i+2} \cup \cdots$ and $k_{i-1}^{2}$ is the 2 -handle used in constructing $A_{i-1}$ (See (\#).) We will show that, for each $i \geq 1$, the inclusion

$$
\Gamma_{i} \cup k_{i-1}^{2} \hookrightarrow N_{i}^{\prime}
$$

is a homotopy equivalence. Hence, $N_{i}^{\prime}$ has finite homotopy type. 
Given $i \geq 1$, let $A_{i}^{\prime}=A_{i} \cup k_{i-1}^{2}$ and $E_{i}^{\prime}=A_{i}^{\prime} \cup B_{i}$. Note that $E_{i}^{\prime}$ is not a subset of $M_{*}^{n}$ since $B_{i}$ is not. We now have a cobordism $\left(E_{i}^{\prime}, \Gamma_{i}^{\prime}, \Gamma_{i}\right)$ where (attaching handles from right to left)

$$
E_{i}^{\prime}=C_{i}^{\prime} \cup h_{i}^{1} \cup h_{i}^{2} \cup k_{i}^{2} \cup k_{i}^{3} \cup k_{i-1}^{2} \approx \Gamma_{i} \times[0,1] \cup k_{i-1}^{2} .
$$

Here the left-hand boundary $\Gamma_{i}^{\prime}$ may be obtained from $\Gamma_{i}$ by performing surgery on a regular neighborhood of a circle representing the element $a_{i} \in \pi_{i}\left(\Gamma_{i}, p_{i}\right)$.

We may reorder handles so that $k_{i-1}^{2}$ is attached first. (Sliding $k_{i-1}^{2}$ past $h_{i}^{2}$, $k_{i}^{2}$ and $k_{i}^{3}$ is standard; attaching $k_{i-1}^{2}$ before $h_{i}^{1}$ requires a quick review of our construction.) Let $\widehat{k}_{i-1}^{2} \subset$ int $\left(k_{i-1}^{2}\right)$ be a small regular neighborhood of the core of $k_{i-1}^{2}$, extended along the product structure of $C_{i}^{\prime}$ to the right-hand boundary $\Gamma_{i}$. See Figure 4(a). Carving from $E_{i}^{\prime}$ the interior of this "thin"

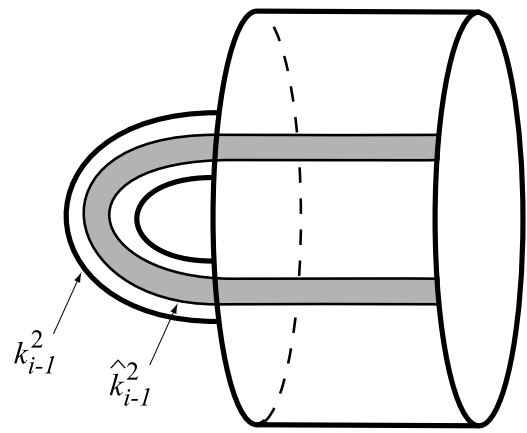

(a) $E_{i}^{\prime} \approx \Gamma_{i} \times[0,1] \cup k_{i-1}^{2}$

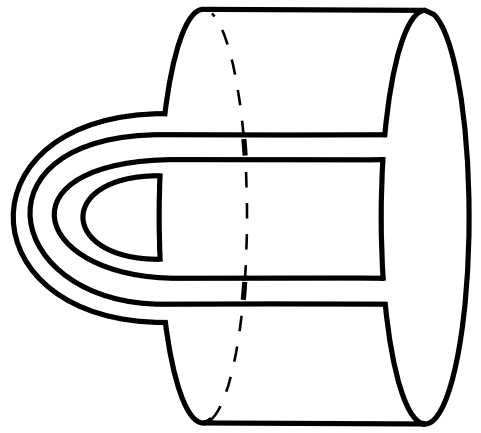

(b) $\left(E_{i}^{\prime \prime}, \Gamma_{i}^{\prime}, \Gamma_{i}^{\prime \prime}\right)$

Figure 4

2-handle $\widehat{k}_{i-1}^{2}$, we obtain a cobordism $\left(E_{i}^{\prime \prime}, \Gamma_{i}^{\prime}, \Gamma_{i}^{\prime \prime}\right)$ where $\Gamma_{i}^{\prime \prime} \approx \Gamma_{i}^{\prime}$ since $\Gamma_{i}^{\prime \prime}$ is obtained from $\Gamma_{i}$ by essentially the same surgery that produced $\Gamma_{i}^{\prime}$. See Figure 4(b). Furthermore, since $A_{i} \cup B_{i} \approx \Gamma_{i} \times[0,1]$, it is easy to see that $E_{i}^{\prime \prime}$ is also a product. The existing handle structure on $E_{i}^{\prime}$, provides a handle decomposition $E_{i}^{\prime \prime}=C_{i}^{\prime \prime} \cup h_{i}^{1} \cup h_{i}^{2} \cup k_{i}^{2} \cup k_{i}^{3}$ where $C_{i}^{\prime \prime}$ is a small product neighborhood of $\Gamma_{i}^{\prime \prime}$. Recalling that $h_{i}^{2}$ was attached along a circle in $\Gamma_{i}$ representing $a_{i+1}^{-2} a_{i}^{-1} a_{i+1} a_{i}$ where $a_{i+1}$ represents a circle that runs once through $h_{i}^{1}$, and noting that (in $\Gamma_{i}^{\prime \prime}$ ) $a_{i}$ has been killed by surgery, we see that $h_{i}^{1}$ and $h_{i}^{2}$ have become a canceling handle pair in $E_{i}^{\prime \prime}$.

We may split $E_{i}^{\prime \prime}$ as $A_{i}^{\prime \prime} \cup B_{i}^{\prime \prime}$ where $B_{i}^{\prime \prime}=C_{i}^{\prime \prime} \cup h_{i}^{1} \cup h_{i}^{2}$ and $A_{i}^{\prime \prime}$ is obtained from the left-hand component of $B_{i}^{\prime \prime}$ by attaching $k_{i}^{2}$ and $k_{i}^{3}$. Alternatively, 
$A_{i}^{\prime \prime}=A_{i}^{\prime}-\operatorname{int}\left(\widetilde{k}_{i-1}^{2}\right)$ where $\widetilde{k}_{i-1}^{2}$ is the interior of a regular neighborhood of the core of $k_{i-1}^{2}$ extended to the right-hand boundary of $A_{i}^{\prime}$. (The 2-handle $\widetilde{k}_{i-1}^{2}$ should be thinner than $k_{i-1}^{2}$, but thicker than $\widehat{k}_{i-1}^{2}$.) It has already been established that $E_{i}^{\prime \prime}$ is a product. Since $h_{i}^{1}$ and $h_{i}^{2}$ form a canceling pair, $B_{i}^{\prime \prime}$ is also a product. Thus, it follows from regular neighborhood theory that

$$
A_{i}^{\prime \prime} \approx \Gamma_{i}^{\prime} \times[0,1] .
$$

This last identity will be key to the remainder of the proof.

Claim For each $i \geq 1, A_{i}^{\prime}$ strong deformation retracts onto $\Gamma_{i} \cup k_{i-1}^{2}$.

Proof of Claim It suffices to show that $\Gamma_{i} \cup k_{i-1}^{2} \hookrightarrow A_{i}^{\prime}$ is a homotopy equivalence. Let $b_{i-1}^{n-2}$ be a belt disk for $k_{i-1}^{2}$ that intersects the thinner 2 handle $\widetilde{k}_{i-1}^{2}$ in a belt disk $\widetilde{b}_{i-1}^{n-2}$. By pushing in from the attaching region of $k_{i-1}^{2}$ we may collapse $\Gamma_{i} \cup k_{i-1}^{2}$ onto $\Gamma_{i}^{\prime} \cup b_{i-1}^{2}$. See Figure 5. Using a similar
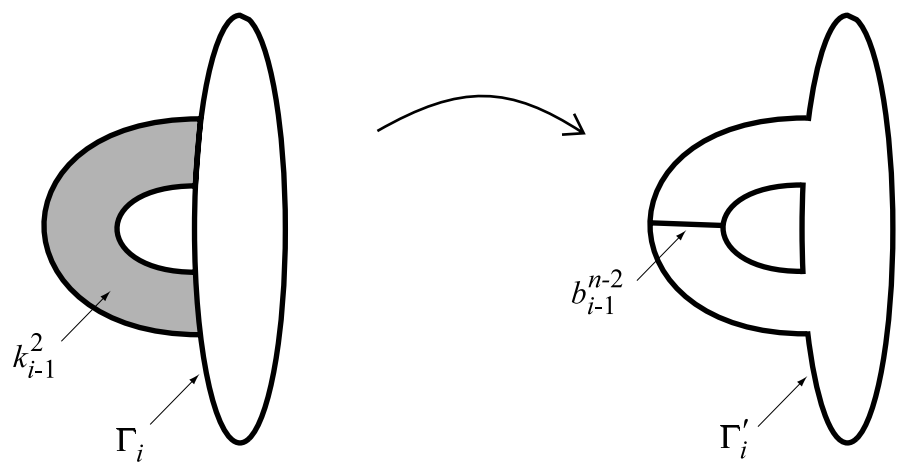

Figure 5

move, we may collapse $A_{i}^{\prime}$ onto $A_{i}^{\prime \prime} \cup \widetilde{b}_{i-1}^{n-2}$. Then, using the product structure on $A_{i}^{\prime \prime}$ we may collapse $A_{i}^{\prime \prime} \cup \widetilde{b}_{i-1}^{n-2}$ onto $\Gamma_{i}^{\prime} \cup b_{i-1}^{n-2}$. Composing the resulting homotopy equivalences shows that $\Gamma_{i} \cup k_{i-1}^{2} \hookrightarrow A_{i}^{\prime}$ is a homotopy equivalence and completes the proof of the claim.

It is now an easy matter to verify $\left.{ }^{(* *}\right)$. Let $i \geq 1$ be fixed. We know that $A_{i}^{\prime}$ strong deformation retracts onto $\Gamma_{i} \cup k_{i-1}^{2}$, and for each $j>i$, we may extend (via the identity) the strong deformation retraction of $A_{j}^{\prime}$ onto $\Gamma_{j} \cup k_{j-1}^{2}$ to a strong deformation retraction of $A_{j-1} \cup A_{j}$ onto $A_{j-1}$. By standard methods, we may assemble these strong deformation retractions to a strong deformation retraction of $N_{i}^{\prime}$ onto $\Gamma_{i} \cup k_{i-1}^{2}$. 


\section{An Open Question}

Our work on pseudo-collars was partly motivated by [3], which the authors advertise as a version of Siebenmann's thesis for Hilbert cube manifolds. Their result provides necessary and sufficient conditions for a Hilbert cube manifold $X$ to be "Z्Z-compactifiable", ie, compactifiable to a space $\widehat{X}$ such that $\widehat{X}-X$ is $\mathcal{Z}$-set in $\widehat{X}$.

Theorem 5.1 (Chapman and Siebenmann) A Hilbert cube manifold $X$ admits a $\mathcal{Z}$-compactification iff each of the following is satisfied.

(a) $X$ is inward tame at infinity.

(b) $\sigma_{\infty}(X)=0$.

(c) $\tau_{\infty}(X) \in \varliminf^{1}\left\{W h \pi_{1}(X \backslash A) \mid A \subset X\right.$ compact $\}$ is zero.

Notice that conditions a) and b) are identical to conditions (1) and (3) of Theorem 1.1. The obstruction in c) is an element of the "first derived limit" of the indicated inverse system, where $W h$ denotes the Whitehead group functor. See [3] for details.

It is not well-understood when conditions a)-c) imply $\mathcal{Z}$-compactifiability for spaces that are not Hilbert cube manifolds. In [8], a polyhedron was constructed which satisfies the hypotheses of Theorem 5.1, but which fails to be $\mathcal{Z}$-compactifiable. However, it is unknown whether a finite dimensional manifold that satisfies these conditions can always be $\mathcal{Z}$-compactified. In trying to answer this question, it seems worth noting that Chapman and Siebenmann employed a two step procedure in proving their result. First they showed that a Hilbert cube manifold satisfying conditions a) and b) is pseudo-collarable. Next they used the pseudo-collar structure, along with condition c) and some powerful Hilbert cube manifold techniques to obtain a $\mathcal{Z}$-compactification.

In contrast with the infinite dimensional situation, the manifolds $M_{*}^{n}$ constructed in this paper satisfy conditions a) and b) yet fail to be pseudo-collarable. Furthermore, an inductive application of the exact sequence on page 157 of [16] shows that each group $G_{i}$ appearing in the canonical inverse sequence representative of $\pi_{1}\left(\varepsilon\left(M_{*}^{n}\right)\right)$ has trivial Whitehead group. It follows that $\tau_{\infty}\left(M_{*}^{n}\right)=0$. Thus, the $M_{*}^{n}$ 's would appear to be ideal candidates for counterexamples to an extension of Theorem 5.1 to the case of finite dimensional manifolds. More generally, we ask:

Question Can a $\mathcal{Z}$-compactifiable open $n$-manifold fail to be pseudo-collarable? 


\section{References}

[1] S Buoncristiano, C P Rourke, B J Sanderson, A Geometric Approach to Homology Theory, LMS Lecture Note Series, Cambridge University Press (1976)

[2] M G Brin, T L Thickstun, 3-manifolds which are end 1-movable, Mem. Amer. Math. Soc. 81 no. 411 (1989)

[3] T A Chapman, L C Siebenmann, Finding a boundary for a Hilbert cube manifold, Acta Math. 137 (1976) 171-208

[4] D E Cohen, Combinatorial Group Theory: a topological approach, London Mathematical Society, Student Texts 14, Cambridge University Press (1989)

[5] M W Davis, Groups generated by reflections and aspherical manifolds not covered by Euclidean space, Ann. of Math. 117 (1983) 293-325

[6] J Dydak, Local n-connectivity of quotient spaces and one point compactification, from: "Shape Theory and Geometric Topology, (Dubrovnik, 1981)", Springer Lecture Notes, 870, Springer, Berlin-New York (1981) 48-72

[7] C R Guilbault, Manifolds with non-stable fundamental groups at infinity, Geometry and Topology 4 (2000) 537-579

[8] CR Guilbault, A non-Z-Compactifiable polyhedron whose product with the Hilbert cube is $\mathcal{Z}$-compactifiable, Fund. Math. 168 (2001) 165-197

[9] C R Guilbault, Compactifications of manifolds with boundary, in progress

[10] A Hatcher, Algebraic Topology, Cambridge University Press, Cambridge, UK (2002)

[11] A Karrass, D Solitar, Subgroups of HNN groups and groups with one defining relator, Canad. J. Math. 23 (1971) 627-643

[12] P Olum, Mappings of manifolds and the notion of degree, Ann. Math. 58 (1953) $458-480$

[13] C P Rourke, B J Sanderson, Block bundles: II. Transversality, Ann. Math. 87 (1968) 256-278

[14] C P Rourke, B J Sanderson, Introduction to Piecewise-Linear Topology, Springer-Verlag, New York, 1982.

[15] L C Siebenmann, The obstruction to finding a boundary for an open manifold of dimension greater than five, Ph.D. thesis, Princeton University, 1965.

[16] F Waldhausen, Whitehead groups of generalized free products, Algebraic Ktheory, II: "Classical algebraic K-theory and connections with arithmetic (Proc. Conf., Battelle Memorial Inst., Seattle Wash., 1972) Lecture Notes in Math., Vol. 342, Springer, Berlin, (1973) 155-179

[17] C T C Wall, Finiteness conditions for $C W$ complexes, Ann. Math. 8 (1965) $55-69$

[18] C T C Wall, (edited by A A Ranicki), Surgery on Compact Manifolds (2 ${ }^{\text {nd }}$ edition), Mathematical Surveys and Monographs, Vol. 69, American Mathematical Society (1999) 